\title{
Electrophysiological Characterization of V2a Interneurons and Their Locomotor-Related Activity in the Neonatal Mouse Spinal Cord
}

\author{
Guisheng Zhong, ${ }^{1}$ Steven Droho, ${ }^{3}$ Steven A. Crone, ${ }^{3}$ Shelby Dietz, ${ }^{1}$ Alex C. Kwan, ${ }^{2}$ Watt W. Webb, ${ }^{2}$ Kamal Sharma, ${ }^{3}$ \\ and Ronald M. Harris-Warrick ${ }^{1}$ \\ ${ }^{1}$ Department of Neurobiology and Behavior and ${ }^{2}$ School of Applied and Engineering Physics, Cornell University, Ithaca, New York 14853, and ${ }^{3}$ Department \\ of Neurobiology, University of Chicago, Chicago, Illinois 60637
}

The V2a class of Chx10-expressing interneurons has been implicated in frequency-dependent control of left-right phase during locomotion in the mouse. We have used the Chx10::CFP mouse line to further investigate the properties and locomotion-related activity of V2a interneurons in the isolated neonatal spinal cord. V2a interneurons can be divided into three classes, based on their tonic, phasic, or delayed-onset responses to step depolarization. Electrical coupling is found only between neurons of same class and helps to synchronize neuronal activity within the class. Serotonin (5-HT) excites isolated tonic V2a interneurons by depolarizing the neurons and increasing their membrane input resistance, with no significant effects on action potential properties, a mechanism distinct from 5 -HT excitation of commissural interneurons. During NMDA-/5-HT-induced locomotor-like activity, patch-clamp recordings and two-photon calcium imaging experiments show that approximately half of V2a interneurons fire rhythmically with ventral root-recorded motor activity; the rhythmic V2a interneurons fired during one half of the cycle, in phase with either the ipsilateral or the contralateral L2 ventral root bursts. The percentage of rhythmically firing V2a interneurons increases during higher-frequency fictive locomotion, and they become significantly more rhythmic in their firing during the locomotor cycle; this may help to explain the frequency-dependent shift in left-right coupling in Chx10::DTA mice, which lack these neurons. Our results together with data from the accompanying paper (Dougherty and Kiehn, 2009) reinforce earlier proposals that the V2a interneurons are components of the hindlimb central pattern generator, helping to organize left-right locomotor coordination in the neonatal mouse spinal cord.

\section{Introduction}

Neural networks in the ventral spinal cord called central pattern generators (CPGs) organize locomotor behavior in the absence of supraspinal and sensory inputs (Kudo and Yamada, 1987; Cazalets et al., 1995; Kjaerulff and Kiehn, 1996; Cowley and Schmidt, 1997; Kremer and Lev-Tov, 1997; Whelan et al., 2000; Kiehn, 2006). Recently, genetic and developmental tools have been developed to help identify the interneurons that comprise the locomotor CPG network, and a number of different genetically defined neuronal types have been investigated (Jessell, 2000; Lanuza et al., 2004; Hinckley et al., 2005; Gosgnach et al., 2006; Wilson et al., 2007; Crone et al., 2008, 2009; Zhang et al., 2008). The V0 interneurons, comprising a large population of inhibitory commissural interneurons (CINs), are necessary to maintain left-right alternation, while the ipsilaterally projecting inhibitory

Received Sept. 29, 2009; revised Nov. 2, 2009; accepted Nov. 6, 2009.

G.Z. and S.A.C. are supported by National Science Foundation Collaborative Grants 0749467 (R.H.-W.) and 0747238 (K.S.). This work was supported by grants from the Brain Research Foundation (K.S.) and the Paralyzed Veterans of America (K.S.) and by National Institutes of Health Grant NS17323 (R.H.-W.). A.C.K. was supported by the Science and Technology Center Program of the National Science Foundation under Agreement No. ECS-9876771. We thank the members of the laboratories of K.S. and R.H.-W. for their input at multiple stages of this study.

Correspondence should be addressed to Dr. Ronald M. Harris-Warrick, Department of Neurobiology and Behavior, Cornell University, W 159 Seeley G. Mudd Hall, Ithaca, NY 14853. E-mail: rmh4@cornell.edu.

DOI:10.1523/JNEUROSCI.4849-09.2010

Copyright $\odot 2010$ the authors $\quad 0270-6474 / 10 / 300170-13 \$ 15.00 / 0$
V1 interneurons regulate the speed of locomotor behaviors (Lanuza et al., 2004; Gosgnach et al., 2006). V3 interneurons help to maintain robust and stable locomotor output (Zhang et al., 2008). These studies provide a framework to study the relationship between electrical properties of individual neurons in each class and their role in generating patterned locomotor activity.

In this paper, we address the properties of V2a interneurons. The V2 population generates two subgroups, of which the V2a group can be detected by its selective expression of the transcription factor Chx10 (Briscoe et al., 2000; Peng et al., 2007). The V2a interneurons are glutamatergic, ipsilaterally projecting neurons and are activated (as seen by activity-dependent c-fos labeling) during fictive locomotion (Al-Mosawie et al., 2007; Lundfald et al., 2007; Crone et al., 2008, 2009). Their role in locomotion has been studied in Chx10::DTA mice, whose V2a interneurons are selectively ablated (Crone et al., 2008, 2009). V2a interneurons are not essential for locomotor-like rhythm generation but play an important role in left-right alternation (Crone et al., 2008). Our previous study showed that the loss of the V2a interneurons affects the left-right phase in a locomotor frequency-dependent manner (Crone et al., 2009). At low locomotor frequencies, the left-right phase is well maintained in some Chx10::DTA mice, while at high frequencies, the left-right phase shifts toward synchronous left-right limb coordination (Crone et al., 2009). How 
the V2a interneurons participate in the locomotor CPG to regulate left-right coordination remains largely unanswered.

In this study, we used the isolated neonatal Chx10::CFP spinal cord to characterize the membrane properties, synaptic interactions, serotonergic modulation, and firing properties of identified V2a interneurons during fictive locomotion evoked by application of NMDA and serotonin $(5-\mathrm{HT})$. We found that these neurons have heterogeneous electrophysiological properties and exhibit electrical coupling only between neurons within the same electrophysiological class. Serotonin excites a majority of the tonically firing Chx10 interneurons. When fictive locomotion is activated, only approximately half of the V2a interneurons fire rhythmically with the ventral root bursts. However, the percentage of rhythmically firing V2a interneurons increases at higher cycle frequencies, and the neurons become more rhythmic in their firing pattern. These results together with data from the accompanying paper (Dougherty and Kiehn, 2009) reinforce earlier proposals that the $\mathrm{V} 2 \mathrm{a}$ interneurons are components of the hindlimb CPG, helping to organize left-right coordination in the neonatal mouse spinal cord.

\section{Materials and Methods}

Generation and care of Chx10::CFP mice. As shown in Figure 1, the Chx10:LNL:CFP targeting vector was constructed by inserting a stop sequence flanked by loxP recombination sites, a Kozak translation initiation sequence (GCCGCCACC), the coding sequence for enhanced cyan fluorescent protein (CFP), and a Simian Virus 40 (SV 40) polyadenylation site into the chx10 locus at the translation initiation site (ATG). Additionally, 176 bases were removed between the SacII restriction sites flanking the ATG start codon in exon 1. The stop sequence is composed of a neomycin resistance cassette, a false translation initiation sequence, a 5' RNA splice donor site, and SV40 polyadenylation sequences. Homologous DNA arms of $4.2 \mathrm{~kb}$ and $4.6 \mathrm{~kb}$ flank the inserted sequences. The targeting construct was introduced into mouse embryonic stem cells (Primogenix) via electroporation. Following selection with G418, homologous recombinants were identified by Southern blot of BglII-digested genomic DNA using a $505 \mathrm{bp} \mathrm{XbaI/PstI} \mathrm{5'} \mathrm{external} \mathrm{probe} \mathrm{(wild-type} \mathrm{frag-}$ ment: $6.4 \mathrm{~kb}$; targeted allele: $5.6 \mathrm{~kb}$ ) and confirmed with a $746 \mathrm{bp}$ SacII/NcoI $3^{\prime}$ internal probe (wild-type fragment: $6.4 \mathrm{~kb}$; targeted allele: $2.8 \mathrm{~kb}$ ).

Chx 10::CFP mice were generated by breeding Chx10:LNL:CFP mice to Protamine-Cre mice. Recombination in the germline of male F1 mice having the Chx10:LNL:CFP and Protamine-Cre alleles removes the LNL cassette in F2 offspring of ICR females to generate heterozygous mice with one recombined Chx10::CFP allele and one wild-type Chx10 allele. These heterozygous mice have normal locomotor behavior, and their neonatal spinal cords generate normal locomotor-like activity during NMDA and serotonin creatinine sulfate (5-HT) application, implying that the loss of one copy of Chx10 allele does not significantly affect the spinal locomotor network. Mice were genotyped by PCR of tail DNA [common forward primer: 5'-GTTTCACCTCTGTCCCTGCAAG-3', wild-type reverse (Rev) primer: 5'-CTCCCGACTGTGACTTTCC-3', CFP Rev primer: 5' -ACGCTGAACTTGTGGCCGT-3'].

Immunohistochemistry. Postnatal day 0 (P0) Chx10::CFP pups were anesthetized and perfused with $4 \%$ paraformaldehyde. Spinal cords were dissected out, postfixed for $2 \mathrm{~h}$ in $4 \%$ paraformaldehyde, and washed overnight in PBS. Tissue was cryoprotected in 30\% sucrose for 2-4 h and mounted in OCT medium (Tissue Tek) for cryosectioning. Transverse sections $(14 \mu \mathrm{m})$ of lumbar spinal cord were stained with antibodies to GFP/CFP (Invitrogen, rabbit, 1:4000) according to the methods described in the study by Crone et al. (2008). Biotinylated secondary antibodies (donkey, 1:500) followed by ABC amplification (Vector Laboratories) and DAB color reaction (Sigma) were used visualize staining. Slides were dehydrated in graded concentrations of ethanol and then xylenes and mounted in Permount media (Fisher Scientific). Images were obtained with a Zeiss Axioplan II microscope and a Zeiss Axiocam digital camera and analyzed with OpenLab 3.1 software (Improvision).
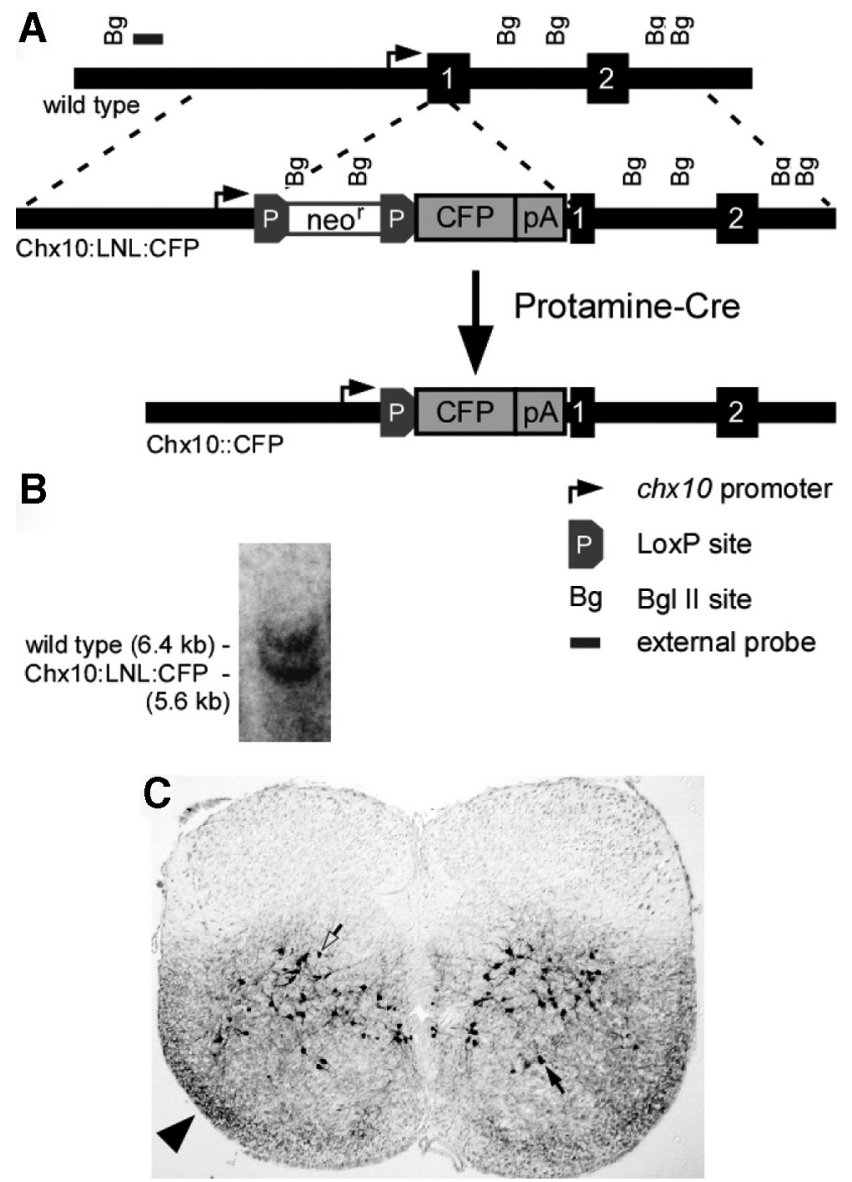

Figure 1. Targeting CFP expression to V2a neurons. $A$, The targeting construct used to insert the coding sequence for CFP and a LoxP-flanked selectable marker (Neor) into exon 1 of the wild-type chx10 locus. The recombined Chx10::CFP allele results from breeding Chx10:LNL:CFP mice to Protamine-Cre mice. $B$, Southern blot of embryonic stem cell DNA demonstrating homologous recombination of the Chx10:LNL:CFP targeting vector into the chx 10 locus. C, Immunohistochemistry using antibodies to CFP labels cell bodies and processes of V2a neurons in the lumbar spinal cord of Chx10::CFP mice. V2a neurons are located in the ventral spinal cord and their axons project within the ventral lateral funiculus (arrowhead). V2a neurons with both large-diameter cell body (filled arrow) and small-diameter cell body (open arrow) are observed.

Whole-cord preparations. Experiments were performed using the isolated spinal cord from neonatal (P1-P4) Chx10::CFP mice. The animal protocol was approved by the Institutional Animal Care and Use Committee at Cornell University and was in accordance with National Institutes of Health guidelines. The dissection was performed as described previously (Zhong et al., 2007). Briefly, neonatal mice were decapitated and eviscerated in cold oxygenated $\left(95 \% \mathrm{O}_{2}\right.$ and $5 \%$ $\mathrm{CO}_{2}$ ) low-calcium Ringer solution composed of the following (in $\mathrm{mm}$ ): $111 \mathrm{NaCl}, 3.08 \mathrm{KCl}, 25 \mathrm{NaHCO}_{3}, 1.18 \mathrm{KH}_{2} \mathrm{PO}_{4}, 3.5 \mathrm{MgSO}_{4}, 0.25$ $\mathrm{CaCl}_{2}$, and 11 glucose. The spinal cord extending from T8/T9 to S3/4 was removed and pinned dorsal side up in a recording chamber superfused with oxygenated Ringer solution composed the following (in mm): $111 \mathrm{NaCl}, 3.08 \mathrm{KCl}, 25 \mathrm{NaHCO}_{3}, 1.18 \mathrm{KH}_{2} \mathrm{PO}_{4}, 1.25 \mathrm{MgSO}_{4}$, $2.52 \mathrm{CaCl}_{2}$, and 11 glucose. All experiments were performed at room temperature $\left(20-23^{\circ} \mathrm{C}\right)$.

Slice preparations. Spinal cords from Chx10::CFP mice were isolated in low-calcium Ringer solution. Transverse spinal cord slices $(350 \mu \mathrm{m})$ were made with a vibrating microtome (Leica Microsystems) and transferred to regular mouse Ringer solution at $30^{\circ} \mathrm{C}$ for $30 \mathrm{~min}$ before recording at room temperature with constant perfusion $(\sim 3 \mathrm{ml} / \mathrm{min})$ of mouse Ringer. CFP-expressing interneurons were identified under epifluorescent illumination and visualized using differential interference contrast optics (Axioskop 2FS plus, Carl Zeiss). Drugs were added by perfusion as described above. 
Patch-clamp recording. V2a interneurons are located along the dorsalventral midline, far from either the ventral or the dorsal surface of the cord (Fig. 1C). The CFP-expressing interneurons could not be visualized from either surface, making it impossible to make whole-cell recordings in the isolated whole spinal cord. To solve this problem, we removed the dorsal half of the L2 segment with fine scissors during the dissection. This allowed us to visualize and record from the L2 V2a interneurons during NMDA-/5-HT-induced fictive locomotion. As originally described by Kjaerulff and Kiehn (1996), we found that the whole cord with the L2 dorsal horn removed is still capable of generating regular fictive locomotion, characterized by left-right and flexor-extensor alternation. Wholecell recordings were made with electrodes pulled from thick-wall borosilicate glass (WPI) on a vertical puller (Narishige) with resistances of 6-10 M $\Omega$, which were lowered through the cut dorsal surface of the L2 segment. The pipette solution contained the following (in mM): 138 K-gluconate, 10 HEPES, 5 ATP-Mg, 0.3 GTP-Li, and $0.0001 \mathrm{CaCl}_{2}(\mathrm{pH}$ 7.4 with $\mathrm{KOH}$ ). The seal resistance obtained before recordings was always $>2$ G $\Omega$. Patch-clamp recordings were made with a Multiclamp 700B amplifier (Molecular Devices) and were driven by Clampex (pClamp 9, Molecular Devices). Data were filtered at $20 \mathrm{kHz}$.

Fictive locomotor activity was evoked by bath application of NMDA $(6-10 \mu \mathrm{M})$ and 5-HT $(6-10 \mu \mathrm{M})$ in normal Ringer solution. Suction electrodes were placed on the ipsilateral L2 (iL2), ipsilateral L5 (iL5), and contralateral L2 (cL2) ventral roots. Rhythmic burst activity from ventral roots was bandpass filtered $(100 \mathrm{~Hz}$ to $1 \mathrm{kHz})$. One hundred percent of control experiments $(n=10)$ made from cords with the dorsal horn removed from the L2 segment showed the expected alternation between ipsilateral and contralateral (iL2-cL2) bursts and between ipsilateral flexor (L2) and extensor (L5) bursts during NMDA- and 5-HT-evoked fictive locomotion. Thus, in many whole-cord preparations with the L2 dorsal horn removed, we monitored fictive locomotion using only one suction electrode on the iL2 ventral root to characterize NMDA-/ 5-HT-induced locomotor-like activity while monitoring V2a interneuron activity with either patch-clamp recording or two-photon calcium imaging. The following drugs were purchased from Sigma: 5-HT, D(-)-2-amino-5-phosphonopentanoic acid (AP-5), 6-cyano-7nitroquinoxaline-2,3-dione disodium salt (CNQX), strychnine, picrotoxin, and kynurenic acid.

Data analysis. Locomotor-like activity was recorded from the intact spinal cord preparation during bath application of NMDA and 5-HT. Clampfit 9.0, Excel, and Spike 2 were used for data analysis. A cycle of motor nerve activity started with the onset of an iL2 ventral root burst and ended at the onset of the next iL2 burst; these onsets were determined by a custom-made program in Spike2 to detect when the rectified signal exceeded the average noise level between bursts by a preset amount. Data were analyzed only in preparations that showed stable rhythmic ventral root activity in iL2 and in which the Chx10 neuron action potential overshoot was $>0 \mathrm{mV}$. Individual $\mathrm{V} 2 \mathrm{a}$ interneurons were recorded for $>20$ min during NMDA- and 5-HT-induced locomotor-like activity. To determine the preferred firing phases of V2a interneurons, the phase of each spike during $>35$ cycles was normalized by the onset of the iL2 burst. A double-normalization process was used (Shefchyk and Jordan, 1985; Zhong et al., 2007; Berkowitz and Stein, 1994), in which the normalized cycle periods were subdivided into 10 bins, the first 5 representing the phase of iL2 bursting and the last 5 equivalent to the cL2 phase (see Fig. $6 B, F$ ). The mean firing rate was established by dividing the number of action potentials in a bin by the duration for at least 35 cycles of activity. The average firing frequencies in each bin were used to create an activity histogram. Circular statistics were used to determine the significance of the phasing of V2a interneurons firing during fictive locomotion (Kjaerulff and Kiehn, 1996; Zar, 2000). The latency to each spike was measured relative to the beginning of the iL2 ventral root burst. The phase of individual spikes was determined by the latency divided by the cycle length. In each V2a interneuron, the phase of $\sim 30$ spikes was measured to plot the mean vector (see Fig. $6 B, F$ ). The direction of a mean vector represents the average preferred phase of firing of the neuron, while its length, $r$, is a measure of statistical significance of the peak preferred phase, indicating the sharpness of the tuning of the spikes around their mean. Vectors with a phase in the range of $0.0-0.5$ corre- sponded to cells firing in phase with iL2. $p$ values for the significance of $r$ were calculated as described by Kjaerulff and Kiehn (1996) and Zar (2000). V2a interneurons were defined as rhythmic if $p<0.05$ by the Rayleigh test.

Two-photon calcium imaging. Cells within whole isolated spinal cords were labeled by bolus injection of Fluo-3 AM calcium indicator dyes as previously described (Stosiek et al., 2003; Garaschuk et al., 2006; Kwan et al., 2009), with one major difference: the dye-injecting pipette was inserted from the cut dorsal surface, with the dorsal surface of the cord at L2 segment removed as described above. Two-photon calcium imaging was performed using a custom-built multiphoton microscope based on a Bio-Rad Radiance 2000 scanning unit, which has been described in detail (Kwan et al., 2009). To detect CFP fluorescence (Wilson et al., 2007), the excitation laser was tuned to $900 \mathrm{~nm}$ and emission of $<500 \mathrm{~nm}$ was collected behind an optical filter (BGG22, Chroma) with a bialkali photomultiplier tube (HC125-02, Hamamatsu). To detect Fluo-3 fluorescence, the excitation laser was tuned to $800 \mathrm{~nm}$ and emission of $>500 \mathrm{~nm}$ was collected behind an optical filter (575/150, Chroma) with a GaAsP photomultiplier tube (H7422, Hamamatsu). This spectral separation scheme is similar to the one described for separating GFP and Fluo-3 fluorescence (Wilson et al., 2007; Kwan et al., 2009). Compared with GFP, a significant portion of the CFP fluorescence emission is $<500 \mathrm{~nm}$ so it is easier to image CFP-expressing neurons in deeper regions. Twophoton calcium imaging data were analyzed by routines written in MATLAB (Mathworks). The time-lapse fluorescence trace of a cell, $F(t)$, was measured by defining the regions of interest, each consisting of one cell with significant Fluo-3 fluorescence, and then summing the pixel values within each region in each image frame. The baseline of the fluorescence trace, $F_{\mathrm{o}}$, was defined to be the mean of $F(t)$ over the first 10 imaging frames. The change in fluorescence, $\mathrm{d} F / F_{\mathrm{o}}$, is defined to be $\left(F(t)-F_{\mathrm{o}}\right) / F_{\mathrm{o}}$. In the presence of transmitters, fictive locomotion is continuous so the baseline fluorescence may be constantly fluctuating; therefore, the calculated $\mathrm{d} F / F_{\mathrm{o}}$ values are only an estimate. To calculate the mean firing phase of a cell, the period of the fictive locomotion, $T$, was calculated by taking the Fourier transform of the ventral root recording and then locating the peak $>0.2 \mathrm{~Hz}$. The raw $\mathrm{d} F / F_{\mathrm{o}}$ was smoothed with a moving average of three imaging frames and the raw ventral root recording was low pass filtered and rectified. Cross-correlation of the processed $\mathrm{d} F / F_{\mathrm{o}}$ and root recording was calculated. The mean phase was calculated by dividing the time of the first cross-correlation peak by $T$, the period of the fictive locomotion. To estimate the significance of the mean phase, we calculated the value of the Fourier transform of $\mathrm{d} F / F_{\mathrm{o}}$ at the period of fictive locomotion, $\mathrm{d} F / F_{\mathrm{o}}(f=1 / T)$, which was then normalized to the largest of such values from all the cells within one experiment. A histogram of these normalized $\mathrm{d} F / F_{\mathrm{o}}(f=1 / T)$ values shows no clear cutoff value so we arbitrarily assign cells to be significantly rhythmic if the normalized $\mathrm{d} F / F_{\mathrm{o}}(f=1 / T)$ value is $>0.25$. We note that this estimate of significance is different from the conventional $r$ values used for plotting circular plots, which carry actual statistical significance, and so should be interpreted accordingly.

Firing properties of isolated V2a interneurons. V2a interneurons were isolated from most rapid synaptic inputs with a combination of AP-5 $(15-20 \mu \mathrm{M})$ and CNQX $(10 \mu \mathrm{M})$ or kynurenic acid (2 mM) to block glutamatergic synapses, picrotoxin $(5 \mu \mathrm{M})$ to block GABAergic synapses, and strychnine $(10 \mu \mathrm{M})$ to block glycinergic synapses in the lumbar spinal cord slices. Some of the neurons fired spontaneously, while others were silent at rest and required depolarizing current pulses to evoke action potentials. To assure uniformity of the measurements, all neurons were held below threshold at $-60 \mathrm{mV}$ with a bias current. To determine the $F-I$ plot, 1 s current injections of increasing amplitude were delivered, and the average spike frequency was determined by counting the number of spikes during the $1 \mathrm{~s} \mathrm{step,} \mathrm{or} \mathrm{instantaneous} \mathrm{frequency} \mathrm{measured} \mathrm{by} \mathrm{the}$ first interspike interval during a step; these were plotted against the injected current amplitude. The slope of the $F-I$ curve was linearly fitted. The voltage threshold for action potential generation was measured as the peak of the second derivative of voltage with time during the rising phase of the action potential. The spike afterhyperpolarization (AHP) amplitude was measured from the action potential threshold to the minimal voltage after the action potential. Additionally, we measured the 


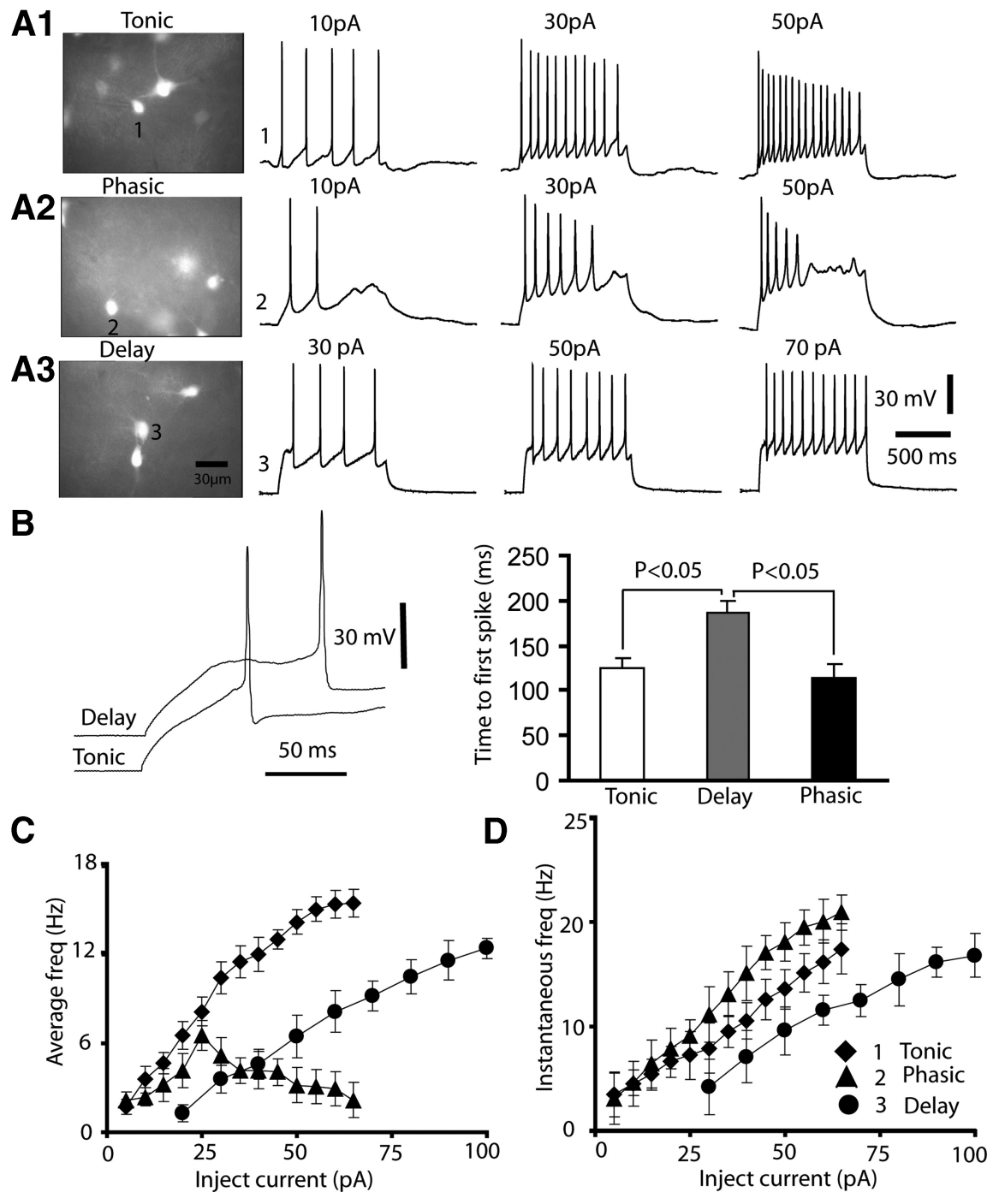

Figure 2. Intrinsic membrane properties of V2a interneurons. $\boldsymbol{A}$, Responses of three different V2a interneurons to a series of increasing depolarizing current pulses from a holding potential of $-60 \mathrm{mV}$. The fluorescent pictures are shown in the left. $\boldsymbol{B}$, Comparing the first spike latencies of delay-like $(30 \mathrm{pA})$ and tonic $(10 \mathrm{pA})$ V2a interneurons when the average frequency is $4 \mathrm{~Hz}$. The average results are shown in the right. $C$, Relationship for the average spike frequency as a function of increasing current amplitude for the three $\mathrm{V} 2 \mathrm{a}$ interneurons from $\boldsymbol{A}$. $\boldsymbol{D}$, Relationship for the instantaneous spike frequency as a function of increasing current amplitude for the three V2a interneurons from $\boldsymbol{A}$.

peak AHP, the minimal voltage after the action potential, with respect to ground. The spike amplitude was measured from the peak AHP to the peak of the action potential. The action potential half-width was established at the voltage halfway from the spike threshold to the peak of the action potential. The minimal amount of current necessary for spike generation was defined as rheobase in our measurements. Input resistance was estimated by averaging the response to small hyperpolarizing current pulses. We used one-way ANOVAs with post hoc Tukey tests to compare the membrane properties of different types of V2a interneurons. The effects of serotonin on the membrane properties were analyzed by paired two-tailed Student's $t$ test. Results were considered statistically significant at $p<0.05$. Data are expressed as mean \pm SD.

\section{Results}

\section{Fluorescent labeling of V2a interneurons}

To visualize V2a interneurons in the spinal cord, transgenic mice from the ICR stain were generated in which V2a interneurons are fluorescently labeled by inserting the coding sequence for $C F P$ into the chx10 locus. Figure $1 A$ shows the targeting construct (Chx10:LNL:CFP) containing the coding sequence for CFP and a
LoxP-flanked selectable marker which blocks expression of CFP. Homologous recombination of the Chx10:LNL:CFP targeting construct in embryonic stem cells was demonstrated by Southern blot (Fig. $1 B$ ). To remove the selectable marker and activate CFP expression in Chx10expressing (V2a) neurons, Chx10::CFP mice (Fig. $1 A$ ) were generated by breeding Chx10:LNL:CFP mice to ProtamineCre mice (see Materials and Methods for details); this results in heterozygous mice with one intact copy of Chx10 and one copy in which the Chx10 gene has been replaced by CFP. In Chx10::CFP mice, the cell bodies and processes of V2a INs can be identified by immunohistochemistry (Fig. 1C) using antibodies to CFP or directly by CFP fluorescence (Fig. $2 \mathrm{~A}$ ).

\section{V2a interneurons show heterogeneity based on intrinsic firing properties} As a first step to examine the intrinsic firing properties of V2a interneurons, we characterized their membrane properties in slice preparations from Chx10::CFP lumbar spinal cords. Electrophysiological recordings of $\mathrm{CFP}^{+} \mathrm{V} 2 \mathrm{a}$ interneurons were performed after isolating them from most synaptic input with blockers of rapid synaptic transmission (glutamate, GABA and glycine). One hundred twenty-one V2 neurons were recorded for the intrinsic membrane properties analysis from 51 newborn (P2-P4) Chx10::CFP mice. V2a interneurons could be divided into three major groups based on their firing responses to depolarizing current steps (Fig. 2A1-A3). Sixty-seven percent of V2a interneurons (82/121) displayed tonic firing throughout a suprathreshold step (Fig. 2A1). Twenty-four percent of V2a interneurons (29/121) fired only phasically: they fired action potentials at the beginning of the depolarizing step, but the spike amplitude quickly dropped and the neurons fell silent for the duration of the current step (Fig. 2A2). The remaining 8\% (10/ 121) of V2a interneurons showed a significant delay at the beginning of the current step before starting to fire tonically (Fig. 2A3). We compared the first spike latency from tonic, phasic and delay V2a interneurons when the average frequency was $4 \mathrm{~Hz}$. Figure $2 \mathrm{~B}$ shows that the delay V2a interneurons had a significantly longer first spike latency. The excitability of the neurons during a series of depolarizing current steps was determined by generating $\mathrm{F}-I$ plots of their average and instantaneous first spike frequencies (Fig. 2C,D). The average and instantaneous $F-I$ curves of the tonic and delay $\mathrm{V} 2 \mathrm{a}$ interneurons both displayed an approximately linear relationship. However, the delay V2a interneurons had a much higher rheobase than both tonic and phasic interneurons (Fig. $2 D$ ) (tonic: $14.8 \pm 7.9 \mathrm{pA}$; phasic: $12.5 \pm 10.1 \mathrm{pA}$; delay: $24.1 \pm 14.3 \mathrm{pA}$; ANOVA; $p<0.005$ ) (Table 1 ). The instantaneous $F-I$ relation for the phasic V2a interneurons showed a linear relationship (Fig. 2D), while its average frequency did not 
Table 1. The membrane properties of three different populations of $\mathrm{V} 2 \mathrm{a}$ interneurons

\begin{tabular}{lcccc}
\hline & $\begin{array}{l}\text { Tonic } \\
(n=82)\end{array}$ & $\begin{array}{l}\text { Phasic } \\
(n=29)\end{array}$ & $\begin{array}{l}\text { Delay-like } \\
(n=10)\end{array}$ & $p$ value \\
\hline Em (mV) & $-46.4 \pm 4.6$ & $-48.7 \pm 5.2$ & $-50.1 \pm 6.3$ & 0.112 \\
Rin (Mf1) & $1034 \pm 243$ & $1603 \pm 378$ & $713 \pm 147$ & 0.008 \\
Cf (pF) & $23.6 \pm 16.2$ & $19.5 \pm 10.4$ & $25.6 \pm 11.8$ & 0.105 \\
Rheobase (pA) & $14.8 \pm 7.9$ & $12.5 \pm 10.1$ & $24.1 \pm 14.3$ & 0.004 \\
AP amplitude (mV) & $26.7 \pm 6.9$ & $21.8 \pm 8.7$ & $23.9 \pm 9.5$ & 0.143 \\
AP half-width (ms) & $2.4 \pm 0.5$ & $3.4 \pm 0.7$ & $2.6 \pm 0.8$ & 0.032 \\
AP threshold (mV) & $-32.2 \pm 4.4$ & $-29.6 \pm 6.8$ & $-27.5 \pm 5.4$ & 0.096 \\
AHP amplitude (mV) & $18.7 \pm 4.1$ & $15.8 \pm 6.1$ & $16.1 \pm 6.4$ & 0.168
\end{tabular}

p values indicate the level of significance of one-way ANOVAs. Results of post hoc Tukey tests are indicated in Results. AP, Action potential; Em, membrane potential; Rin, input resistance; $\mathrm{Cf}$, membrane capacitance.

vary linearly with the injected current amplitude, reflecting the inability to fire continuously during high current steps (Fig. 2C).

We further analyzed the membrane properties of the three classes of V2a interneurons (Table 1). All three classes had similar resting potentials; however, significant differences were observed in the input resistance of each neuron class (Table 1) (ANOVA; $p<0.01$ ). Phasic V2a interneurons had a significantly higher input resistance than tonic and delay V2a interneurons (Tukey tests; $p<0.01$ ). The low input resistance of delay V2a interneurons is consistent with the high rheobase in these neurons. Finally, we analyzed the action potential properties of V2a interneurons, including the amplitude, half-width, threshold, and AHP amplitude. These were for the most part very similar, but the spike half-width varied significantly across cell types (Table 1) (ANOVA; $p<0.05$ ). Phasic V2a interneurons had a wider action potential half-width than both tonic and delay neurons (Tukey tests; $p<0.05)$.

Because the hyperpolarization-activated inward current $\left(I_{\mathrm{h}}\right)$ is often found in neurons participating in rhythmic neural networks (Angstadt and Calabrese, 1989; McCormick and Pape, 1990; Lüthi et al., 1998; Thoby-Brisson et al., 2000; HarrisWarrick, 2002; Grillner, 2003), we examined whether this current is found in the three classes of V2a interneurons. Evidence for $I_{\mathrm{h}}$ is detected in current-clamp recordings by a depolarizing sag voltage during a maintained hyperpolarizing current step, and a depolarizing rebound at the end of the step. Both phasic and tonic V2a interneurons displayed a variety of different responses to subthreshold current steps. Thirty-eight percent of tonic V2a interneurons $(31 / 82)$ displayed little or no sag voltage $(<2 \mathrm{mV}$ at $-120 \mathrm{mV}$ ) or rebound potentials (Fig. $3 A$, top). Sixty-two percent of tonic V2a interneurons (51/82) had sag voltages and rebound potentials when measured at the minimum voltage around $-120 \mathrm{mV}$ (Fig. $3 A$, middle and bottom). The sag amplitude grew with increasing hyperpolarization and was related to the rebound potential amplitude (Fig. $3 B$ ). In approximately a third of all tonic interneurons (27/82), the rebound potential passed the action potential threshold and generated spikes (Fig. $3 A$, bottom). With regard to the phasic V2a interneurons, we found that $90 \%(26 / 29)$ had detectable sag voltages and postinhibitory rebound potentials when measured at the minimum voltage at around $-120 \mathrm{mV}$. The sag amplitude grew with increasing hyperpolarizations, as did the associated rebound potentials. In $28 \%$ of the phasic V2a interneurons with sag potentials $(6 / 26)$, the rebound potential passed the action potential threshold and generated spikes. The remaining $10 \%$ of phasic neurons $(3 / 29)$ had no detectable sag voltages or postinhibitory rebound potentials. Neurons in the delay interneuron group con-
A
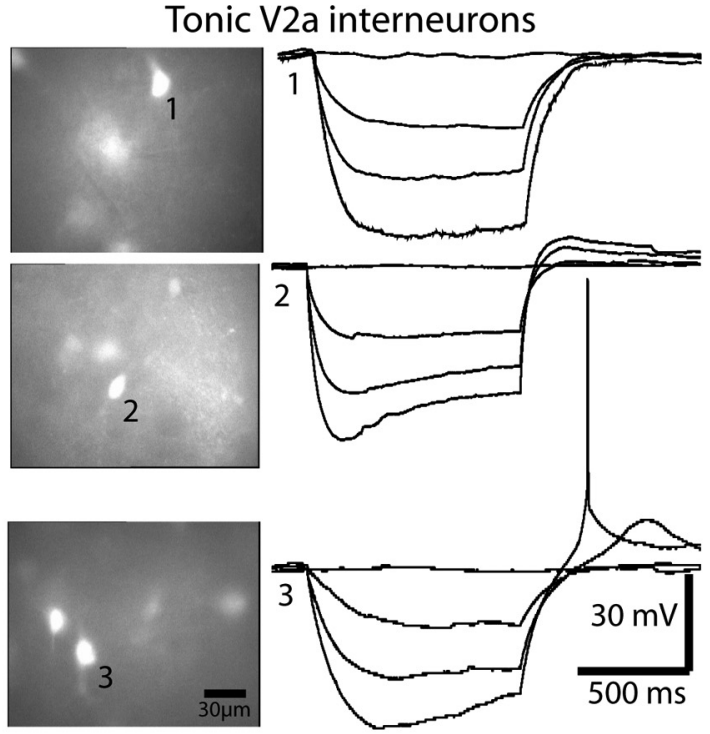

B

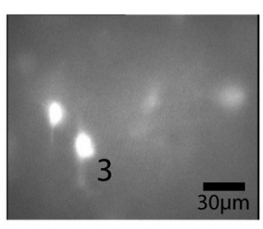

Tonic V2a interneuron-2

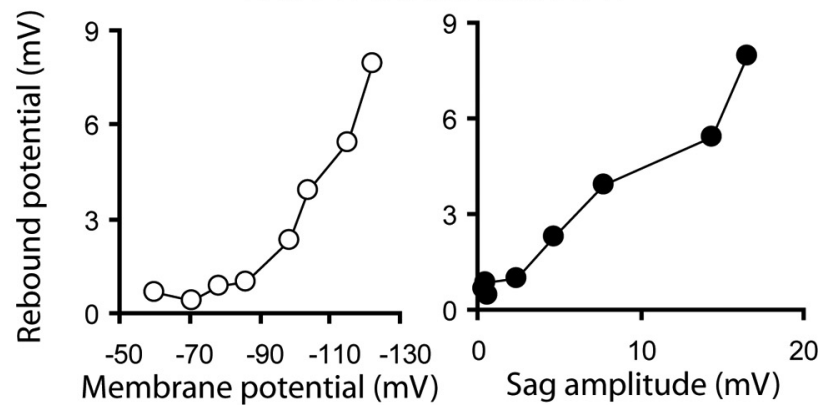

Figure 3. Responses of tonic V2a interneurons to hyperpolarizing current steps. $\boldsymbol{A}$, The response to increasing hyperpolarizing $1 \mathrm{~s}$ current pulses from three tonic V2a interneurons with most fast synaptic transmission blocked. The fluorescent pictures are shown in the left. $\boldsymbol{B}$, Left, Relationship between the rebound potential and minimum membrane potential; right, relationship between rebound potential and sag amplitude from V2a interneuron.

sistently displayed sag voltages and rebound potentials when measured at the minimum voltage around $-120 \mathrm{mV}$, but none of them rebounded sufficiently to fire an action potential. Thus, the majority of V2a interneurons have an h-current-induced sag voltage and postinhibitory rebound, but there are members of both tonic and phasic classes that do not express $I_{\mathrm{h}}$.

\section{Electrical coupling between V2a interneurons of the same class}

We used dual whole-cell recordings in slice preparations prepared from neonatal Chx10::CFP lumbar spinal cords to investigate whether V2a interneurons form electrical synapses with other V2a interneurons. These experiments were performed in the presence of blockers of glutamate, GABA and glycine (kynurenate, picrotoxin, strychnine) in the bath to eliminate potential chemical synapses. We found that when the two recorded V2a interneurons were in the same electrophysiological class (i.e., tonic or phasic), $29 \%$ of recorded pairs (16/54) were electrically coupled. Figure $4 A$ shows an example of electrical coupling between two phasic V2a interneurons, in which injection of either depolarizing or hyperpolarizing current into one neuron resulted in a smaller de- or hyperpolarization in the second neuron. The coupling was reciprocal and of similar strength in both directions, showing that this was a nonrectifying junction (Fig. 4A). Three of 7 pairs of phasic V2a interneurons showed detectable 


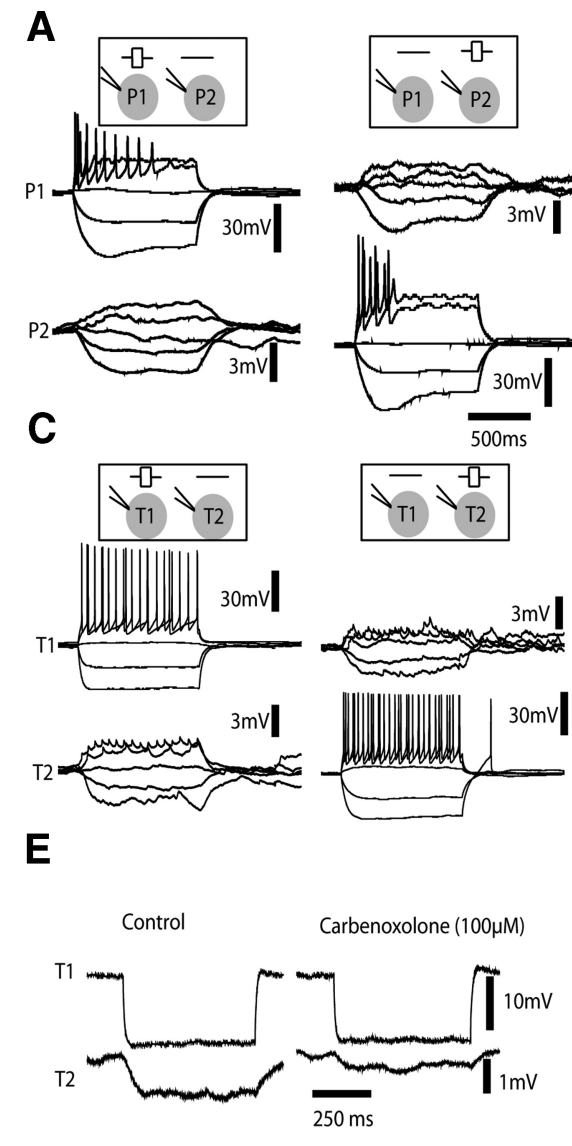

B
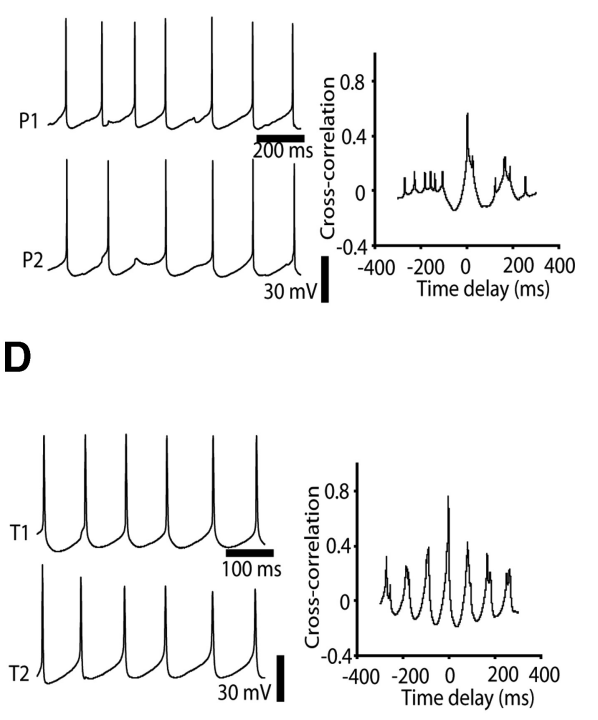

$\mathbf{F}$

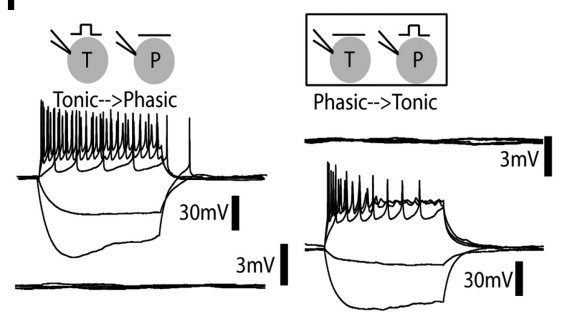

Figure 4. Electrical coupling between V2a interneurons with similar membrane properties. $\boldsymbol{A}$, Left, Responses of phasic V2a interneuron-1 (P1) to a series of current injections while the activity of the phasic P2 was monitored. Right, Responses of phasic P2 to a series of current injections while the activity of the phasic $\mathrm{P} 1$ was monitored. $\boldsymbol{B}$, Left, When both neurons were depolarized above action potential threshold with bias current, they tended to fire action potentials synchronously. Right, Cross-correlogram of data taken from left trace. There was a positive peak at time 0 . C, Left, Responses of tonic V2a interneuron T1 to a series of current injections while the activity of the tonic $\mathrm{T} 2$ was monitored. Right, Responses of $\mathrm{T} 2$ to a series of current injections while the activity of T1 was monitored. $\boldsymbol{D}$, Left, When both neurons were depolarized above action potential threshold with bias current, they tended to fire action potentials synchronously. Right, Cross-correlogram of data taken from left trace. $\boldsymbol{E}$, The electrical coupling between a different tonic pair was significantly reduced by $100 \mu \mathrm{m}$ carbenoxolone. $\boldsymbol{F}$, Left, Responses of tonic V2a interneuron 1 to a series of current injections while the activity of the phasic interneuron-2 was monitored. Right, Responses of phasic V2a interneuron-2 to a series of current injections while the activity of the tonic interneuron-1 was monitored. No coupling was detected.

electrical coupling. The mean coupling coefficient from recorded phasic pairs was $0.13 \pm 0.02(n=3$ pairs $)$. When tonically depolarized above threshold, the two phasic V2a interneurons fired action potentials synchronously; the cross-correlogram shows a strong peak near $0 \mathrm{~ms}$ and evenly spaced smaller peaks indicative of the regular firing of these neurons (Fig. $4 B$ ).

Similar results are seen with pairs of tonic V2a interneurons Twenty-eight percent (13/47) of tonic V2a interneuron pairs displayed detectable electrical coupling in slice preparations. The coupling was reciprocal and of similar strength in both directions, as we found in phasic pairs. The mean coupling coefficient from tonic pairs was $0.14 \pm 0.03$ ( $n=13$ pairs). As found in phasic V2a pairs, these coupled tonic V2a interneurons fired synchronous action potentials when tonically depolarized above action potential threshold (Fig. 4D). Since these measurements were made from slice preparations, which could eliminate longer connections that leave the slice, the fraction of coupled neurons could be even higher. Electrical coupling between V2a interneurons was significantly reduced by bath application of the gap junction blocker, $100 \mu \mathrm{M}$ carbenoxolone, while the input resis- tance did not change (Fig. 4E) $(n=3)$. The delay V2a interneurons are sufficiently rare that we did not find any pairs to measure electrical coupling within this class.

We have not observed electrical coupling in any pair of $\mathrm{V} 2 \mathrm{a}$ interneurons from different electrophysiological classes. For example, if one neuron fired phasically while the other neuron fired tonically during depolarizing current pulses, no coupling was observed (Fig. $4 F)$ ( $n=7$ pairs). Additionally, we never detected any fast chemical synapses between V2a interneurons when the antagonists of glutamate, GABA and glycine receptors were left out of the Ringer solution ( $n=61$ pairs). However, these results do not completely exclude the possibility of chemical synaptic transmission between V2a interneurons in vivo, since we used slice preparations that could result in a loss of neuronal processes and synapses. Overall, our results indicate the existence of electrical synapses among V2a interneurons with similar electrophysiological properties, which would help to synchronize the activity within each class.

\section{Serotonin increases the excitability of V2a interneurons}

Serotonin (5-HT) plays an important role in enabling the generation of the locomotor rhythm in rodents (MacLean et al., 1998; Madriaga et al., 2004; Christie and Whelan, 2005; Liu and Jordan, 2005; Pearlstein et al., 2005). Thus, we examined the effects of bath-applied 5-HT on the firing properties of tonic V2a interneurons in neonatal mice. To maintain all cells below threshold before application of 5-HT, the membrane potential was adjusted to $-60 \mathrm{mV}$ with bias current injection. Sixty-seven percent of tonic V2a interneurons (14/21) were reversibly depolarized $(7.3 \pm 3.2 \mathrm{mV})$ by application of $10 \mu \mathrm{M}$ 5-HT (data not shown) in the presence of fast synaptic blockers. Serotonin evoked small membrane perturbations in $24 \%$ of V2a interneurons $(5 / 21)$, which are likely to be truncated action potentials from electrically coupled neurons (data not shown).

Next we investigated the effects of 5-HT on the excitability of V2a interneurons in the presence of blockers of fast synaptic transmission. In these experiments, all the V2a interneurons were of the tonic firing type. Serotonin increased the excitability of $61 \%$ of recorded V2a interneurons (11/18). Figure $5 A$ shows a typical response of one tonic V2a interneuron to a series of increasing amplitude current steps before and during serotonin application. Holding currents were adjusted to maintain the membrane potential at $-60 \mathrm{mV}$ between steps in the different conditions. Serotonin increased the number of action potentials per step in the presence of synaptic transmission blockers.

We quantified the responses of 18 tonic V2a interneurons to current steps which evoked $\sim 10 \mathrm{~Hz}$ firing responses under control conditions. In the presence of 5-HT, the firing frequency 
significantly increased in these neurons (control: $9.7 \pm 0.6 \mathrm{~Hz}$; 5-HT: $13.1 \pm 1.24$ $\mathrm{Hz} ; n=18 ; p<0.001$ ) (Fig. $5 A$ ). We generated $F-I$ plots of the average and instantaneous first spike frequency over a range of current step amplitudes; serotonin caused an upward shift in the frequency response to the current steps (Fig. $5 B$ ). As can be seen from the $F-I$ plots, the rheobase value for spike generation was significantly reduced by serotonin from $26.4 \pm$ 12.5 to $11.8 \pm 12.6 \mathrm{pA}(p<0.001)$, but there was no significant change in the slope of the $\mathrm{f} / \mathrm{I}$ relationship (control: $238 \pm 17 \mathrm{~Hz} / \mathrm{nA}$; 5-HT: $246 \pm 23 \mathrm{~Hz} / \mathrm{nA}$; $n=18 ; p>0.05)$.

We measured the effects of 5 -HT on subthreshold responses of V2a interneurons. The input resistance, as measured by the voltage response to a small hyperpolarizing current step from a constant holding potential of $-60 \mathrm{mV}$, was increased by $11 \%$ during $10 \mu \mathrm{M} 5$-HT application (control: $1023 \pm 243 \mathrm{M} \Omega$; 5-HT: $1139 \pm 189 \mathrm{M} \Omega ; n=18 ; p<0.05$ ) (Fig. $5 C, E)$. During prolonged hyperpolarizing steps, 5-HT had no effect on the depolarizing sag potential amplitude, but it did increase the rate of $\mathrm{V} 2 \mathrm{a}$ interneuron firing rebound to fire an action potential. During 5-HT application, the time latency to the rebound action potential was shortened by $12 \pm 7 \%(n=4 ; p<0.05)$ ) (Fig. $5 D)$. 5-HT did not significantly change the amplitude of the rebound potential before reaching action potential threshold, implying that its main effect was on the acceleration of onset of the rebound action potential rather than any effect on the h-current.

Finally, we looked for effects of 5-HT on individual action potential properties, including amplitude, half-width, threshold and afterhyperpolarization. In contrast to our earlier work on 5-HT modulation of commissural interneurons (Zhong et al., $2006 \mathrm{a}$ and b), we did not detect any statistically significant effects of 5-HT on action potential parameters of $\mathrm{V} 2 \mathrm{a}$ interneurons.

\section{Firing patterns of V2a interneurons during NMDA- and 5-HT-induced locomotion}

Previous work has shown that the ablation of V2a interneurons in Chx10::DTA mice markedly affects locomotor patterns in neonatal and adult mice (Crone et al., 2008, 2009). However, whether these neurons are rhythmically active during locomotion was not determined. To answer this question, we recorded the activity of CFP-expressing V2a interneurons from Chx10::CFP neonatal mouse spinal cords during fictive locomotion. These neurons are located in a band along the dorsal-ventral midline, and are not readily accessible from the surface (Crone et al., 2008). Accordingly, we removed the dorsal half of the spinal cord from the L2 segment which allowed us to visualize the CFP-expressing Chx10
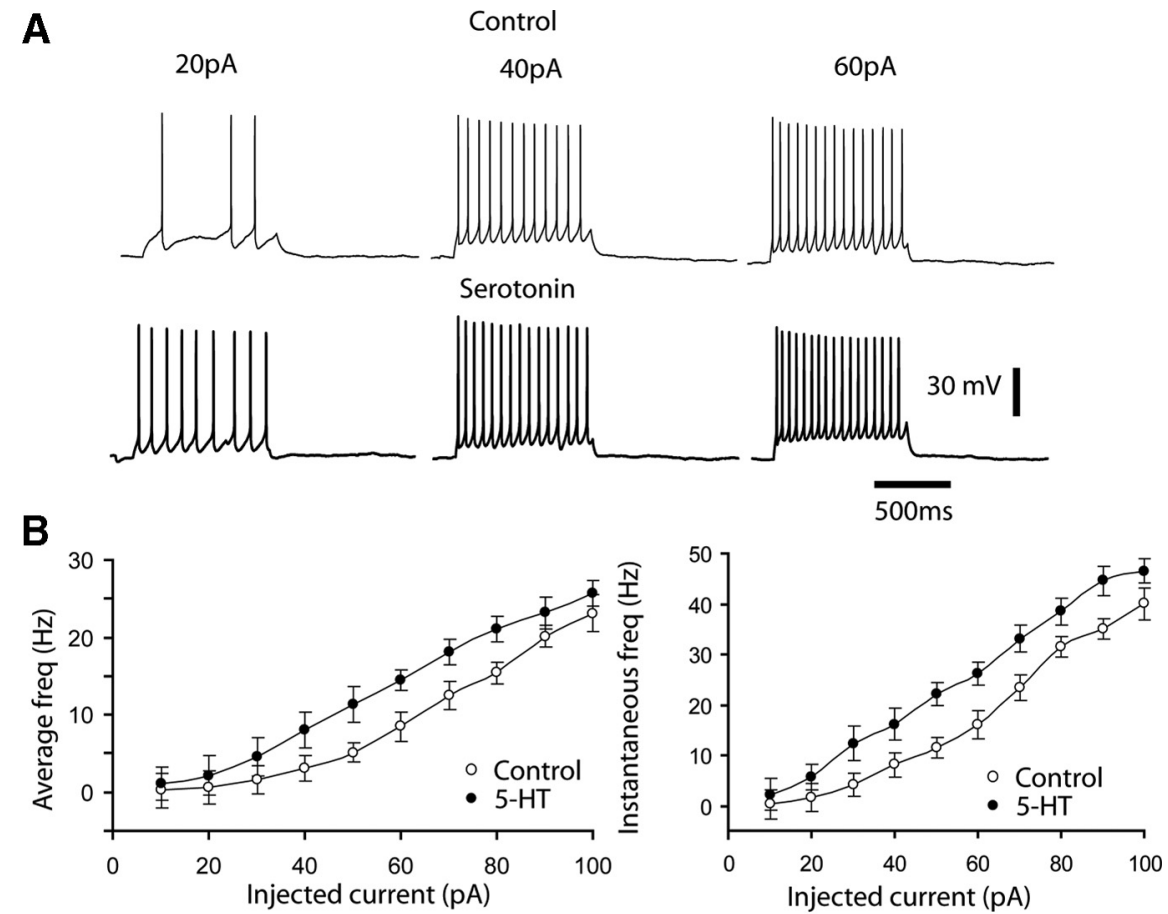

D

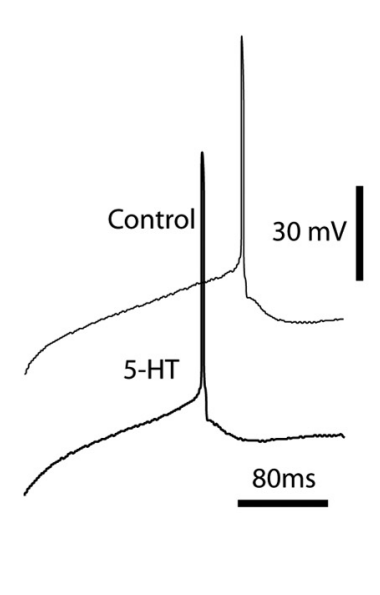

Figure 5. 5-HT increases the excitability of V2a interneurons. $A$, Responses of a V2a interneuron to three depolarizing current steps during control conditions and in the presence of 5-HT (10 $\mu \mathrm{M})$ with most fast synaptic transmission blocked. $\boldsymbol{B}$, Effects of 5-HT on the $\mathrm{F}$-I relationship in V2a interneurons. $C$, Responses of a V2a interneuron to a series of hyperpolarizing current steps from C. $E$, Responses of a V2a interneuron to $-5 \mathrm{pA}$ hyperpolarization pulse before and during 5-HT application, showing an increase in input resistance with 5 -HT.

neurons (Fig. 6A). The locomotor CPG is located in the ventral half of the cord, and fictive locomotor-like activity can be induced by superfusion of the ventral half of the cord with 5-HT and NMDA (Kjaerulff and Kiehn, 1996). These transmitters evoked a typical pattern of left-right ventral root burst alternation, as well as alternation between the ipsilateral L2 (flexordominated) and L5 (extensor-dominated) ventral roots in this L2-dorsal horn-removed preparation.

We found that the V2a interneurons were not homogeneous in their firing patterns during fictive locomotion induced by 5-HT and NMDA. Forty-four percent of V2a interneurons (34/ 78) showed significantly rhythmic activity, while the remaining $56 \%$ (44/78) were either tonically active or silent during fictive locomotion. In the example shown in Figure $6 A$, the V2a interneuron fired rhythmically, with bursts of action potentials in 
A
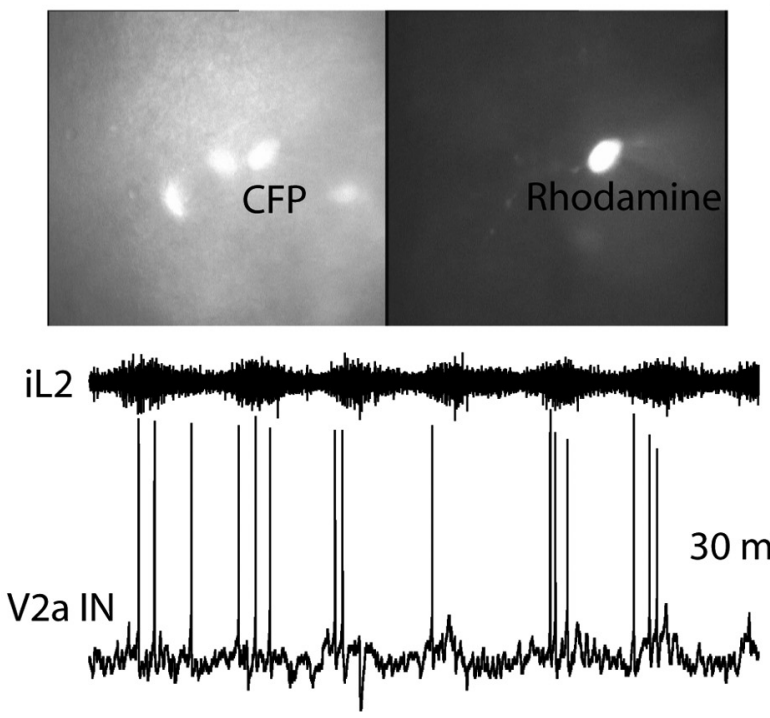

B
E
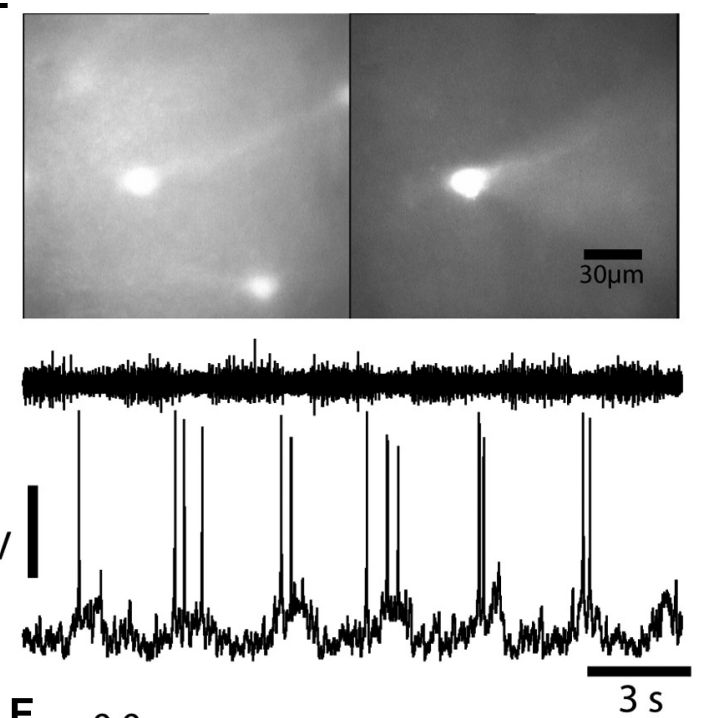
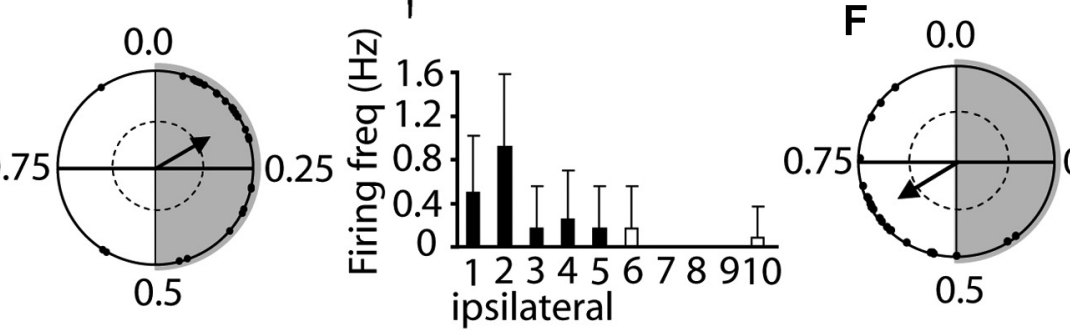

Nㅗㄴ

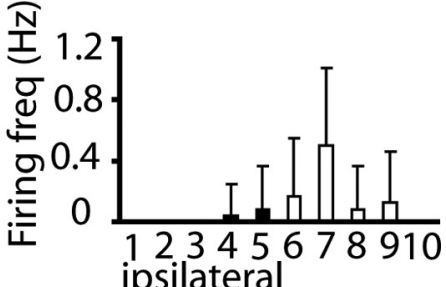

C Voltage clamp $(\mathrm{Vh}=-60 \mathrm{mV})$

iL2
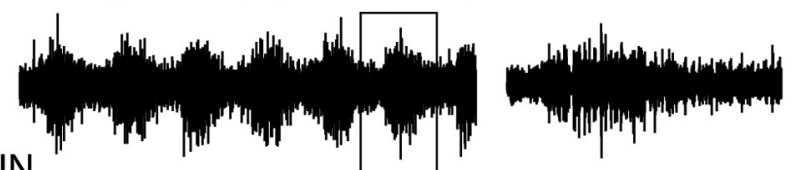

V2a IN

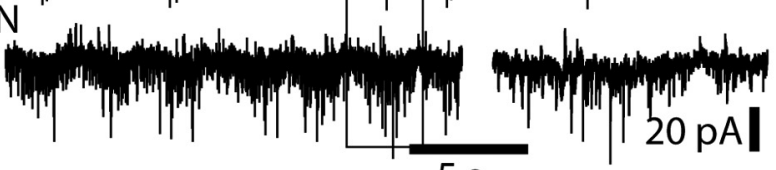

D

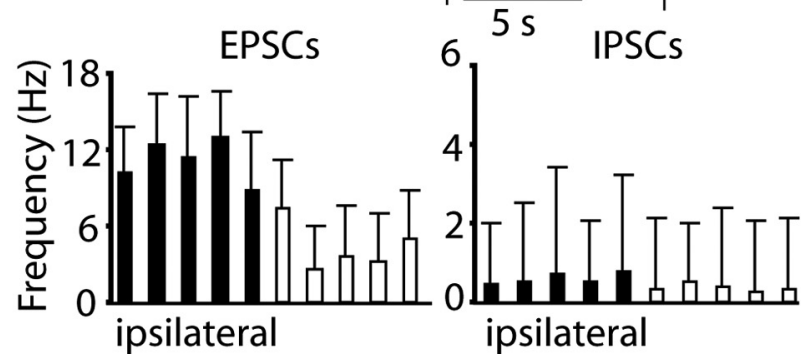

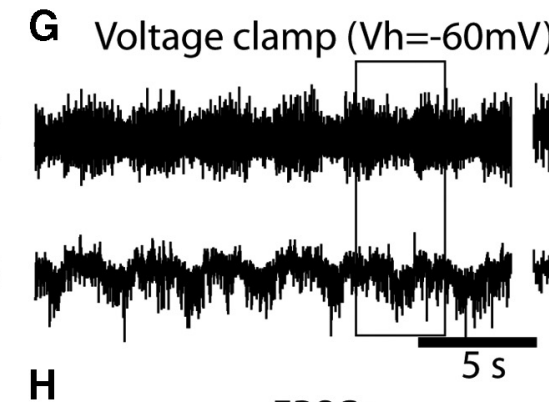

$\mathrm{H}$

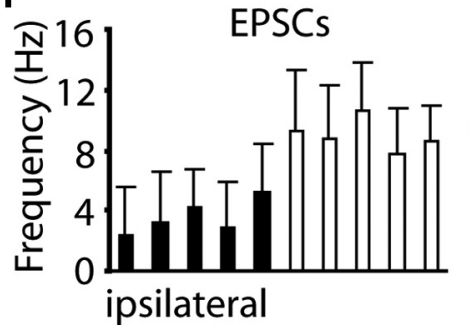

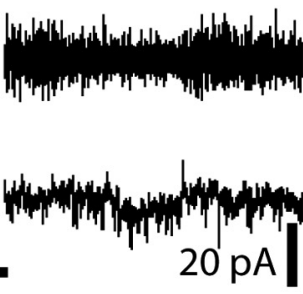

IPSCS

Figure 6. Firing patterns of V2a interneurons during locomotor-like activity. $\boldsymbol{A}$, Intracellular recording from a rhythmic V2a interneuron firing in phase with iL2 ventral root bursts during NMDAand 5 -HT-induced fictive locomotion. Identification of the fluorescent neurons is shown on top. $\boldsymbol{B}$, Circular plot analysis showing that the V2a interneuron from $\boldsymbol{A}$ fired rhythmically during the iL2 firing phase; this is confirmed by a histogram comparing mean firing frequency of the cell in $\boldsymbol{A}$ in relation to the normalized locomotor cycle phase. $\boldsymbol{C}$, During voltage-clamp recording, the V2a interneuron from $\boldsymbol{A}$ received bursts of EPSCS, in phase with iL2 ventral root burst. The right traces show the time from the open box. $\boldsymbol{D}$, Histogram of EPSC and IPSC frequency as a function of normalized cycle period. The axis is the bin number. The first five bins represent the iL2 ventral root activity and the remaining five show the rest of one cycle. EPSC frequency increased during the ipsilateral phase while IPSC frequency was lower and did not vary during the cycle period. $\boldsymbol{E}$, Intracellular recording from a rhythmic V2a interneuron firing out of phase from iL2 VR bursts during NMDA- and 5-HT-induced fictive locomotion. The fluorescent neurons are pictured on the top. $\boldsymbol{F}$, Circular plot analysis showed that the V2a interneuron from $\boldsymbol{E}$ fired rhythmically during the $\mathrm{CL} 2$ firing phase; this is confirmed by the histogram comparing mean firing frequency of the cell in $\boldsymbol{E}$ in relation to the normalized locomotor cycle phase. $\boldsymbol{G}$, Voltage-clamp recording shows that the V2a interneuron from $\boldsymbol{A}$ received bursts of EPSCs, out of phase from the iL2 ventral root burst. The traces to the right were from the open box. $\boldsymbol{H}, \mathrm{A}$ histogram of EPSC and IPSC frequency as a function of normalized cycle period. EPSC frequency increased during the contralateral phase while IPSC frequency was low and did not vary during the cycle.

phase with the ipsilateral L2 ventral root burst. Circular plot analysis (Fig. 6B) showed that the neuron fired broadly throughout the ipsilateral L2 burst, with a mean vector of activity in the first half of the iL2 burst. When the membrane potential was hyper- polarized by current injection, the membrane oscillation appeared to be driven by excitatory synaptic inputs that were correlated to the iL2 ventral root burst. This was verified by voltage-clamp recordings (Fig. $6 C$ ). This V2a interneuron re- 

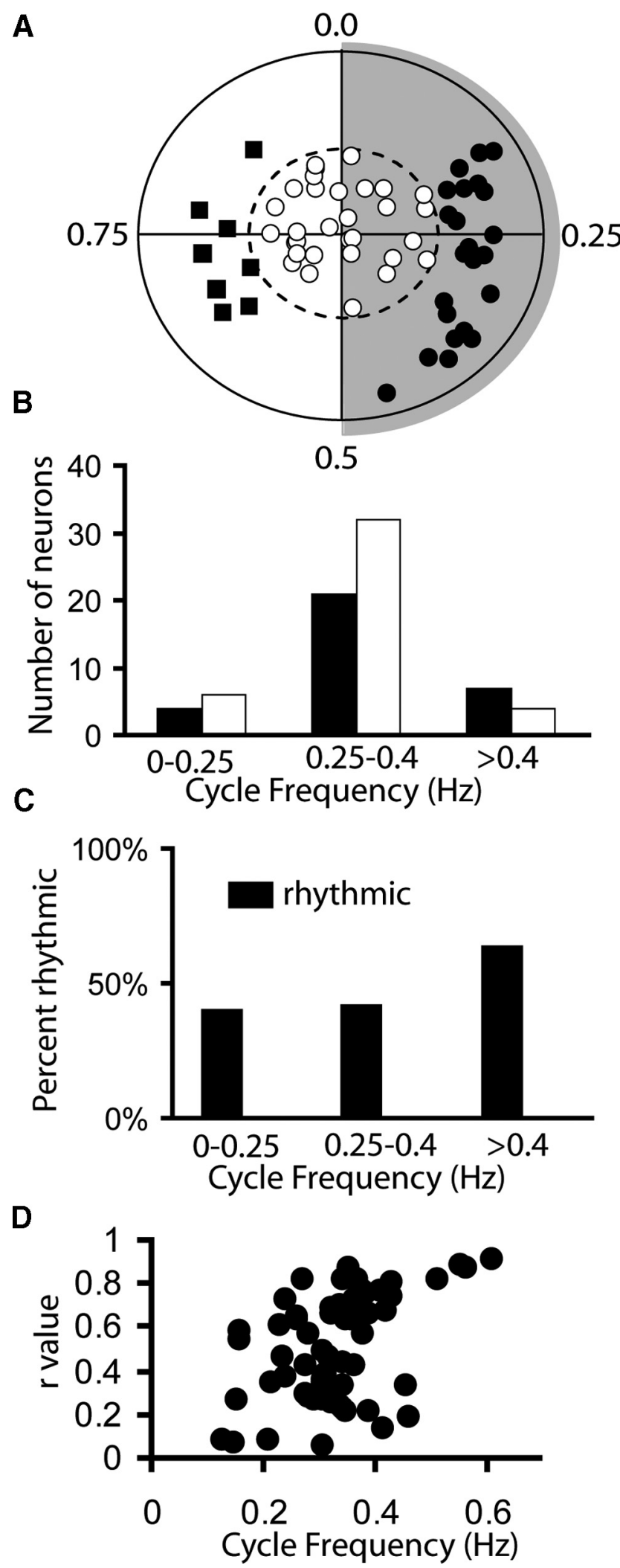

Figure 7. V2a interneuron phasing and speed-dependent rhythmicity during fictive locomotion. $\boldsymbol{A}$, Circular plot showing the mean phase of all the active V2a interneurons during NMDA-/5-HT-induced fictive locomotion ( $n=65$, silent neurons are not included). Each point represents the tip of the vector of mean activity for one V2a interneuron. The direction of the vector represents the preferred phase of firing of the neuron, and the distance from the center indicates the statistical significance of the rhythmicity of the neuron. Black circles represent rhythmically active V2a interneurons with peak activity in phase with iL2 VR bursts; black squares show rhythmic V2a interneurons with peak activity in antiphase with iL2 VR bursts; ceived periodic bursts of EPSCs in phase with the iL2 ventral root bursts (represented by the first five bins) when the membrane potential was held at $-60 \mathrm{mV}$ (Fig. $6 C, D$ ). This neuron received few IPSCs, and these were not temporally correlated with the motor pattern (Fig. 6D); the recordings were repeated at a holding potential of $-45 \mathrm{mV}$ to facilitate IPSC detection, but there was still no rhythmic inhibition of this cell. All recorded rhythmic V2a interneurons were driven predominantly by phasic bursts of EPSPs. Seventy-six percent of the rhythmically active V2a interneurons (26/34) fired in phase with the iL2 ventral root activity.

The remaining $24 \%$ (8/34) of the rhythmically active V2a interneurons fired in antiphase with iL2 ventral root activity, with broad patterns of activity throughout the contralateral ventral root burst. Figure $6 E$ illustrates one example of a rhythmically active V2a interneuron, with bursts of action potentials out of phase from the iL2 ventral root bursts. When the mean phase of activity was calculated by circular plot analysis, this V2a interneuron showed a peak near the center of the CL2 ventral root burst, again reflecting its broad firing throughout the contralateral burst. When the membrane potential was hyperpolarized by current injection, the membrane oscillation was inversely correlated to the iL2 ventral root burst, with apparent bursts of EPSPs depolarizing the neuron during the CL2 burst phase. During voltage-clamp recording at $-60 \mathrm{mV}$, this $\mathrm{V} 2$ a interneuron received periodic bursts of EPSCs in phase with the cL2 ventral root burst (Fig. 6G,H). This V2a interneuron did not receive phasic inhibition, as seen by the relatively uniform and low frequency of IPSCs throughout the cycle (Fig. $6 H$ ); this was also confirmed at $-45 \mathrm{mV}$. These neurons all appeared to be driven primarily by bursts of EPSPs in phase with the contralateral ventral root bursts.

Figure $7 A$ shows a summary in circular plot form of the rhythmic activity of all the $\mathrm{V} 2 \mathrm{a}$ interneurons we recorded $(n=65)$, except for silent neurons. Rhythmic V2a interneurons were active in two groups, with peaks of activity near the center of either iL2 or cL2 bursts. The rhythmic V2a interneurons were active throughout their half of the locomotor cycle. All three electrophysiologically defined classes of V2a INs (phasic, tonic, delay) were capable of firing rhythmically during fictive locomotion. However, we did not record enough V2a interneurons to further characterize the relationship between membrane properties and rhythmicity.

In our previous study demonstrating the effects of ablating V2a interneurons in Chx10::DTA mice, we found that Chx10::DTA mice show a frequency-dependent change in locomotor behavior (Crone et al., 2009). They walk normally at low speeds on a treadmill, with normal alternation of left and right hindlimbs; however, as the treadmill speed was accelerated, the walking was disrupted and eventually replaced by a synchronous "galloping" gait with synchronous movements of the left and right hindlimbs at high speeds. A similar frequency-dependent switch from left-right alternation toward left-right synchrony was observed in the isolated spinal cord (Crone et al., 2009). This suggested the possibility that the V2a interneurons may be selectively

$\leftarrow$

open circles represent nonrhythmic V2a interneurons. The dotted circle indicates the level of statistical significance of the rhythmicity $(p<0.05)$. Gray-shaded areas represent the ipsilateral L2 flexor phase. $\boldsymbol{B}, A$ histogram comparing the number of rhythmic (black) and nonrhythmic (white) V2a interneurons during fictive locomotion at different cycle frequencies. C, Histogram comparing the percentage of rhythmic V2a interneurons during fictive locomotion at different cycle frequencies. $\boldsymbol{D}$, Relationship between the cycle frequency and the rhythmicity of V2a interneurons, as determined by their $r$ values in the circular statistics analysis. 

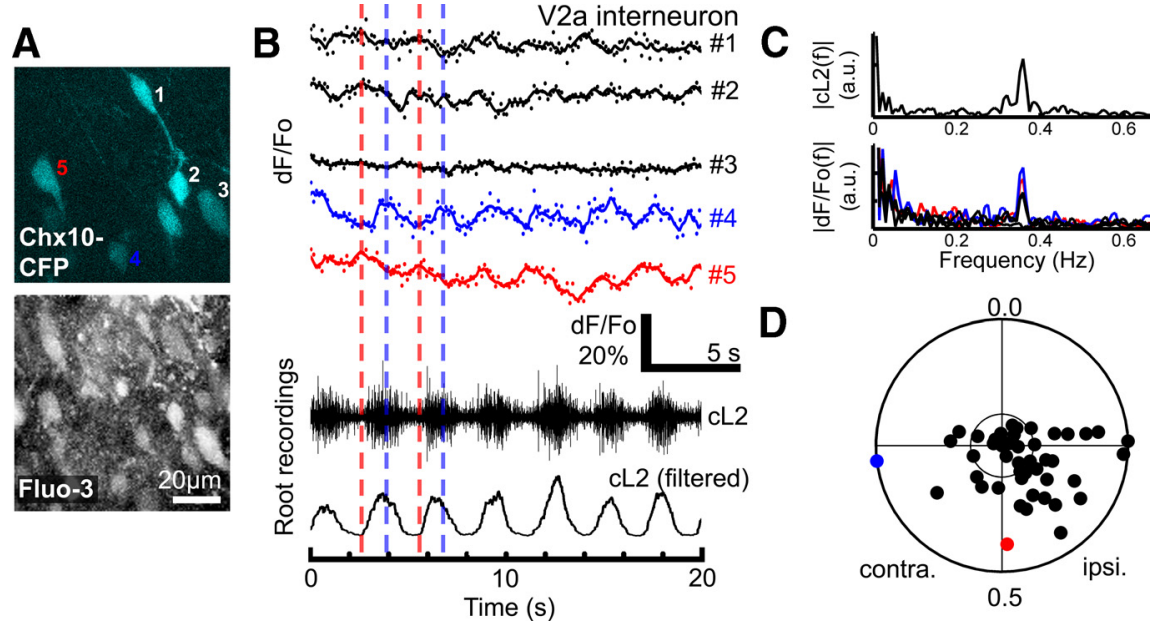

Figure 8. Two-photon calcium imaging of V2a interneurons. $A$, CFP and Fluo-3 fluorescence can be spectrally separated to identify V2a interneurons in a whole in vitro spinal cord with the dorsal horn removed at L2. The CFP panel is a maximal projection stack $10 \mu \mathrm{m}$ thick, whereas the Fluo-3 panel is a single image, so some of the CFP-expressing neurons appear to have no Fluo-3 fluorescence. $\boldsymbol{B}$, Calcium transients of multiple V2a interneurons during fictive locomotion elicited by $9 \mu \mathrm{m} 5-\mathrm{HT}$ and $9 \mu \mathrm{m} \mathrm{NMDA}$. Both the raw data (dots) and the low-pass-filtered fluorescence trace (lines) are displayed and compared with the locomotor cycle from the contralateral L2 VR. Some V2a interneurons are rhythmically active $(1,2,4$, and 5$)$ whereas others are silent (3). Neighboring V2a interneurons can have different peak phases of activity relative to motor output. C, Fourier transforms of the ventral root recording and the five fluorescence traces from $\boldsymbol{B}$. Distinct peaks at the same location suggest that both the ventral root recording and the calcium transients are rhythmic with the same periodicity. $\boldsymbol{D}$, Mean phases of $48 \mathrm{~V} 2 \mathrm{a}$ interneurons were estimated by calculating the cross-correlation between the fluorescence traces and the ventral root recording. Each dot represents one V2a interneuron. Distance from the origin reflects the normalized peak value of the Fourier transform of the fluorescence trace, i.e., from $C$.

more active at high speeds to drive the commissural interneurons that maintain left-right alternation, while another, as yet unidentified, pathway may play the major role in controlling alternation at low speeds. To test this idea, we measured the rhythmicity of $\mathrm{V} 2 \mathrm{a}$ interneurons during fictive locomotion as a function of the cycle frequency. Bath application of 5HT- and NMDA-evoked locomotor activity in the neonatal spinal cord with somewhat different cycle frequencies in different spinal cords. We arbitrarily defined fictive locomotion at $<0.25 \mathrm{~Hz}$ as slow locomotion, from 0.25 to $0.4 \mathrm{~Hz}$ as medium locomotion and $>0.4 \mathrm{~Hz}$ as high speed locomotion. As shown in Figure 7B, we found that V2a interneurons could be either rhythmic or nonrhythmic at all these frequency ranges. Thus, V2a interneurons are not silent at low cycle frequencies and are not exclusively recruited at high cycle frequencies in neonatal mouse spinal cords.

We investigated whether the ratio of rhythmic to nonrhythmic V2a interneurons is different at different cycle frequencies of fictive locomotion. As shown in Figure 7C, only $40 \%$ of V2a interneurons were rhythmic at low or intermediate frequencies; however, at $>0.4 \mathrm{~Hz}$, this percentage increased to $64 \%$. We also tested whether the cycle frequency was related to the rhythmicity of V2a interneurons by looking for a correlation between the cycle frequency of the preparation and the rhythmicity of the recorded V2a interneuron; this was determined by its $r$ value from the circular statistical analysis. The $r$ value reflects the tightness of clustering of spikes during the cycle; neurons that fire diffusely over one half of the cycle will be significantly rhythmic, but with a lower $r$ value than those that fire reliably at a fixed point in the cycle. As illustrated by Figure $7 D$, there was a significant positive correlation between the cycle frequency and $r$ value $(r=0.13+1.11 f ; r=0.42 ; p<0.001 ; n=65)$. Thus, a larger percentage of $\mathrm{V} 2 \mathrm{a}$ interneurons are rhythmic during high-frequency fictive locomotion than at low or intermediate frequencies, and these neurons show more tightly regulated rhythmic activity as the frequency increases.

Two-photon calcium imaging of Chx10 interneurons during fictive locomotion To investigate the spatial relationship between rhythmic and nonrhythmic Chx10 interneurons during fictive locomotion, we used two-photon calcium imaging to record the activity of multiple neurons within the in vitro spinal cord. Twophoton microscopy (Denk et al., 1990; Zipfel et al., 2003) enables deep-tissue imaging, and in the neonatal mouse spinal cord, cells expressing fluorescent proteins can be visualized up to a depth of $\sim 200$ $\mu \mathrm{m}$ (Kwan et al., 2009). As described above, the V2a interneurons are located more deeply than this in the neonatal spinal cord, necessitating removal of the dorsal surface of the L2 segment of the cord. However, recording from cells located deep beneath the cut dorsal surface alleviates concerns about possible damage resulting from the dorsal cut used to expose the V2a interneurons. We have previously shown via combined whole-cell recording and two-photon calcium imaging that large calcium peaks reflect suprathreshold activity of mouse spinal interneurons (Kwan et al., 2009), so calcium imaging can reveal the spatial relationships between cells with different mean phases of activity.

In the whole in vitro spinal cord with the L2 dorsal surface removed, CFP-expressing V2a interneurons were clearly visible from the dorsal surface (Fig. $8 \mathrm{~A}$ ). Multiple bolus injections of the calcium indicator dye Fluo-3 AM resulted in the labeling of many cells in the injection sites. Near the dorsal surface, many Fluo-3labeled cells emitted extremely bright fluorescence, indicating poor cell health; however, more than $\sim 50 \mu \mathrm{m}$ beneath the cut surface, the labeling pattern was similar to that observed in intact whole in vitro spinal cord preparations without any cuts (Kwan et al., 2009). During fictive locomotion elicited by NMDA and 5HT, some V2a interneurons were silent, whereas others displayed rhythmic calcium transients with periodicity similar to the rhythmic motor output recorded from the ipsilateral ventral root in the same lumbar segment (Fig. $8 B$ ). As shown in Figure $8 C$, rhythmic neurons can be identified by sharp peaks at the same frequency (near $0.4 \mathrm{~Hz}$ ) in the Fourier transform of the calcium fluorescence signals and ventral root recordings (Fig. 8C). Direct comparison between neighboring rhythmic V2a interneurons within the same field of view revealed that neighboring cells could have significantly different phases relative to the motor output. There was also no obvious spatial grouping of V2a interneurons that were active or silent during fictive locomotion.

To characterize the mean phases of V2a interneuron activity, we computed the cross-correlation between the fluorescence traces and the ventral root recordings. The significance of a mean phase was then evaluated by calculating the value of the Fourier transform of the fluorescence trace at the period of fictive locomotion as measured from the ventral root bursts (Fig. $8 C$, peak values near $0.4 \mathrm{~Hz}$, for example). From $48 \mathrm{~V} 2 \mathrm{a}$ interneurons, the mean phases and their significances distributed widely when 
graphed on a circular plot (Fig. $8 D$ ). Strongly rhythmic V2a interneurons had peaks of activity from $\sim 90^{\circ}$ to $270^{\circ}$, relative to the beginning of the ipsilateral ventral root burst. Thus, different V2a interneurons were rhythmically active during the peak of both the ipsilateral and the contralateral ventral root activity. As was seen with whole-cell recordings, $77 \%$ of the significantly rhythmic V2a interneurons were active during the ipsilateral phase $(24 / 31)$ as opposed to the contralateral phase $(23 \% ; 7 / 31)$. These results reinforce our data with whole-cell recording, and show that a significant percentage of the V2a interneurons are rhythmically active with either the ipsilateral or contralateral ventral root bursts during fictive locomotion. The spatial intermixing of rhythmic, tonically firing, and inactive V2a interneurons in this preparation suggests that there is no clear anatomical distribution of these neurons in the neonatal mouse spinal cord.

\section{Discussion}

Our present study adds evidence to our previous studies (Crone et al., 2008, 2009) that the spinal V2a interneurons, characterized by their expression of Chx10, participate in the CPG (defined as the network driving both rhythm generation and pattern formation) for locomotion in the neonatal mouse spinal cord. To better understand the role of the V2a interneurons in the locomotor CPG, we have here characterized their electrophysiological properties, electrical coupling and serotonin responsiveness, and their firing pattern during fictive locomotion in the newborn Chx10::CFP mouse spinal cord.

\section{Distinct populations of V2a interneurons in the neonatal mouse spinal cord}

We found that the V2a interneurons are not a homogeneous class. We identified three major classes of V2a interneurons, which fire tonically, phasically, or with a significantly delayed onset during depolarizing current steps. Within each major class of V2a interneurons, subpopulations could be further defined (Dougherty and Kiehn, 2009). All three major classes include neurons showing a hyperpolarization-induced sag component, but there was heterogeneity in each class, and not all tonic or phasic neurons showed evidence of $I_{\mathrm{h}}$. There were also differences in threshold and spike properties between classes. We do not yet know whether these different types of V2a interneurons have different functions in locomotor behavior, but all three classes can be rhythmically active during fictive locomotion.

As final evidence for separate subclasses of V2a interneurons, we detected a significant amount of electrical coupling during paired V2a interneuron recordings, but only within their class; thus, tonic V2a interneurons show coupling to other tonic V2a interneurons but not to phasic V2a interneurons, and vice versa. The coupling coefficients were large enough to synchronize the spike activity of the coupled V2a interneurons when they were both active, suggesting a physiological role to coordinate neuronal activity within each class. Our results are in agreement with previous studies showing that electrical coupling plays an important role in generation of the locomotor pattern in the neonatal rodent spinal cord (Tresch and Kiehn, 2000; Personius et al., 2007). However, in the accompanying article, Dougherty and Kiehn (2009) did not detect electrical coupling between V2a interneurons in either slice or whole-cord preparations. There are several possible reasons which could lead to these differing results. First, different mouse strains with distinct genetic manipulations were used in the two studies (Richter et al., 2009). Our line was generated in ICR strains, while Dougherty and Kiehn used a line based on FVB/N-Swiss Webster mice. Our Chx10::CFP line was generated by homologous recombination to replace one copy of Chx10 with CFP. Dougherty and Kiehn used a BAC-based vector, inserted into an unknown location in the genome. An alternative methodological explanation for the discrepancies that our paired recordings were made with fast synaptic transmission blocked, while Dougherty and Kiehn recorded V2a pairs without synaptic blockers. The lower input resistance and chemical synaptic inputs could make the detection of weak electrical coupling more difficult in the absence of synaptic blockers.

\section{Modulation of V2a interneurons by serotonin}

Serotonin plays a central role to enable locomotor rhythm generation in many species (MacLean et al., 1998; Schmidt and Jordan, 2000; Zhang and Grillner, 2000; Brustein et al., 2003; Madriaga et al., 2004; Liu et al., 2005, 2009; Pearlstein et al., 2005). To help understand how this occurs, we studied the effects of 5-HT on the membrane properties of $C h x 10:: C F P$ interneurons.

Our whole-cell recordings show that 5-HT directly depolarizes and excites the majority of tonic V2a interneurons. 5-HT did not affect the spike properties, but it increased the membrane input resistance by $\sim 10 \%$; 5 -HT also shortened the postinhibitory rebound spike latency, which cannot be explained by the increase of input resistance. One possibility is that 5-HT may reduce a low threshold $\mathrm{I}_{\mathrm{A}}$-type current which normally slows the rate of repolarization after an action potential. In the accompanying paper (Dougherty and Kiehn, 2009), 5-HT was reported to decrease the $\mathrm{R}_{\text {in }}$ and thus increased the rheobase of V2 neurons, but their measurements were made without fast synaptic transmission blocked, and during coapplication of 5-HT with NMDA, and sometimes DA, which depolarize the neurons and enhance synaptic inputs. This makes it difficult to isolate the direct effects of 5-HT on the V2 interneurons but gives a better idea of how the firing properties of these neurons change under conditions of fictive locomotion.

Interestingly, 5-HT excites CINs by a very different mechanism, with no effect on input resistance; instead, it changes the properties of action potentials to allow these neurons to fire at higher frequencies and with a lower rheobase (Zhong et al., 2006a and $b$ ). Thus, the effects of 5-HT are not uniform on all spinal interneurons, but vary with the interneuron type.

\section{Locomotor-related rhythmic firing of V2a interneurons}

A previous study gave anatomical evidence that V2a interneurons synapse on and excite the functionally inhibitory CIN system, including the V0 interneurons (Crone et al., 2008). An activitylabeling study showed that V2a interneurons are activated during locomotion in adult mouse spinal cord (Al-Mosawie et al., 2007), but their pattern of activity could not be determined.

Our whole-cell recording and calcium imaging results show that a significant percentage of V2a interneurons fire rhythmically during fictive locomotion. They are broadly active during one half of the cycle, in phase or antiphase with iL2 root activity. On average, though, over half of the V2a interneurons were not rhythmic in the isolated spinal cord preparation.

We previously showed (Crone et al., 2009) that mice lacking $\mathrm{V} 2 \mathrm{a}$ interneurons display normal alternating locomotor movements at low treadmill speeds, but this is disrupted at intermediate speeds and eventually replaced by left-right synchronous "hopping" at higher treadmill speeds. Similar results were seen during fictive locomotion in the isolated spinal cord. We hypothesized that the V2a-V0 system is the predominant regulator of left-right alternation at high locomotor speeds, but is either silent or redundant with another unidentified functionally inhibitory 
CIN pathway at low speeds. We recorded V2a interneurons' activity in the isolated spinal cord with only the L2 dorsal segment removed which can generate locomotor like activity with a broad range of cycle frequencies during NMDA and 5-HT application. When we correlated rhythmic activity with cycle frequency, we found immediately that a subset of V2a interneurons do fire rhythmically during fictive locomotion at low frequencies. Thus, the simplest hypothesis, that the V2a interneurons are silent at low frequencies but specifically recruited at high frequencies, is not correct. However, the percentage of rhythmically firing V2a interneurons was higher at high frequencies (64\%) than at lower frequencies (40\%). V2a neurons also showed significantly higher rhythmicity at higher frequencies, as measured by their larger $r$ values from the circular statistical analysis; this suggests that they are clustering their action potentials into a smaller portion of the cycle, which could enhance synaptic facilitation and temporal summation in their follower cells. These results support the hypothesis that the V2a neurons may play a greater role in the rhythmic CPG output at higher frequencies than lower frequencies.

We do not know whether the same or different neurons, or classes of V2a neurons, are active at different speeds, as we have not yet been able to manipulate locomotor frequency in a single spinal cord with the dorsal horn-removed to allow individual V2a interneuron recording at different cycle frequencies. In the zebrafish spinal cord, McLean et al. $(2007,2008)$ showed that neurons expressing the Chx10 homolog, alx, comprise several classes that are differentially active at low and high swim speeds. Ventrally located alx-expressing neurons are more active at lower speeds, and reduce or cease firing at higher speeds, while dorsally located alx-expressing neurons are silent at low speeds and preferentially recruited at higher speeds. Whether this differentiation is evolutionarily conserved in mice will require more experimentation under conditions in which we can independently alter the cycle frequency in a single spinal cord.

In summary, our results show that V2a interneurons are heterogeneous in their firing properties. The majority of tonic V2a interneurons are directly excited by serotonin, which helps enable the onset of fictive locomotion. A subset of the V2a interneurons fire rhythmically during NMDA- and 5-HT-induced locomotor-like activity. The percentage of rhythmically firing V2a interneurons increases with high cycle frequencies, and these neurons become more rhythmic in their firing at higher speeds; this may help to explain the frequency-dependent locomotor deficit in the Chx10::DTA mice (Crone et al., 2009). Our results together with data from the accompanying paper (Dougherty and Kiehn, 2009) reinforce earlier suggestions that the V2a interneurons are components of the hindlimb CPG, helping to organize left-right coordination during high-frequency fictive locomotion in the neonatal mouse spinal cord.

\section{References}

Al-Mosawie A, Wilson JM, Brownstone RM (2007) Heterogeneity of V2derived interneurons in the adult mouse spinal cord. Eur J Neurosci 26:3003-3015.

Angstadt JD, Calabrese RL (1989) A hyperpolarization-activated inward current in heart interneurons of the medicinal leech. J Neurosci 9:2846-2857.

Berkowitz A, Stein PS (1994) Activity of descending propriospinal axons in the turtle hindlimb enlargement during two forms of fictive scratching: phase analyses. J Neurosci 14:5105-5119.

Briscoe J, Pierani A, Jessell TM, Ericson J (2000) A homeodomain protein code specifies progenitor cell identity and neuronal fate in the ventral neural tube. Cell 101:435-445.
Brustein E, Chong M, Holmqvist B, Drapeau P (2003) Serotonin patterns locomotor network activity in the developing zebrafish by modulating quiescent periods. J Neurobiol 57:303-322.

Cazalets JR, Borde M, Clarac F (1995) Localization and organization of the central pattern generator for hindlimb locomotion in newborn rat. J Neurosci 15:4943-4951.

Christie KJ, Whelan PJ (2005) Monoaminergic establishment of rostrocaudal gradients of rhythmicity in the neonatal mouse spinal cord. J Neurophysiol 94:1554-1564.

Cowley KC, Schmidt BJ (1997) Regional distribution of the locomotor pattern-generating network in the neonatal rat spinal cord. J Neurophysiol 77:247-259.

Crone SA, Quinlan KA, Zagoraiou L, Droho S, Restrepo CE, Lundfald L, Endo T, Setlak J, Jessell TM, Kiehn O, Sharma K (2008) Genetic ablation of V2a ipsilateral interneurons disrupts left-right locomotor coordination in mammalian spinal cord. Neuron 60:70-83.

Crone SA, Zhong G, Harris-Warrick R, Sharma K (2009) In mice lacking V2a interneurons, gait depends on speed of locomotion. J Neurosci 29:7098-7109.

Denk W, Strickler JH, Webb WW (1990) Two-photon laser scanning fluorescence microscopy. Science 248:73-76.

Dougherty K, Kiehn O (2010) Firing and cellular properties of V2a interneurons in the rodent spinal cord. J Neurosci 30:24-37.

Garaschuk O, Milos RI, Konnerth A (2006) Targeted bulk-loading of fluorescent indicators for two-photon brain imaging in vivo. Nat Prot $1: 380-386$.

Gosgnach S, Lanuza GM, Butt SJ, Saueressig H, Zhang Y, Velasquez T, Riethmacher D, Callaway EM, Kiehn O, Goulding M (2006) V1 spinal neurons regulate the speed of vertebrate locomotor outputs. Nature 440:215-219.

Grillner S (2003) The motor infrastructure: from ion channels to neuronal networks. Nat Rev Neurosci 4:573-586.

Harris-Warrick RM (2002) Voltage-sensitive ion channels in rhythmic motor systems. Curr Opin Neurobiol 12:646-651.

Hinckley CA, Hartley R, Wu L, Todd A, Ziskind-Conhaim L (2005) Locomotor-like rhythms in a genetically distinct cluster of interneurons in the mammalian spinal cord. J Neurophysiol 93:1439-1449.

Jessell TM (2000) Neuronal specification in the spinal cord: inductive signals and transcriptional codes, Nat Rev Genet 1:20-29.

Kiehn O (2006) Locomotor circuits in the mammalian spinal cord. Annu Rev Neurosci 29:279-306.

Kjaerulff O, Kiehn O (1996) Distribution of networks generating and coordinating locomotor activity in the neonatal rat spinal cord in vitro: a lesion study. J Neurosci 16:5777-5794.

Kremer E, Lev-Tov A (1997) Localization of the spinal network associated with generation of hindlimb locomotion in the neonatal rat and organization of its transverse coupling system. J Neurophysiol 77:1155-1170.

Kudo N, Yamada T (1987) N-methyl-D,L-aspartate-induced locomotor activity in a spinal cord-hindlimb muscles preparation of the newborn rat studied in vitro. Neurosci Lett 75:43-48.

Kwan AC, Dietz SB, Webb WW, Harris-Warrick RM (2009) Activity of Hb9 interneurons during fictive locomotion in mouse spinal cord. J Neurosci 29:11601-11613.

Lanuza GM, Gosgnach S, Pierani A, Jessell TM, Goulding M (2004) Genetic identification of spinal interneurons that coordinate left-right locomotor activity necessary for walking movements. Neuron 42:375-386.

Liu J, Jordan LM (2005) Stimulation of the parapyramidal region of the neonatal rat brain stem produces locomotor-like activity involving spinal 5-HT7 and 5-HT2A receptors. J Neurophysiol 94:1392-1404.

Liu J, Akay T, Hedlund PB, Pearson KG, Jordan LM (2009) Spinal 5-HT7 receptors are critical for alternating activity during locomotion: in vitro neonatal and in vivo adult studies using 5-HT7 receptor knockout mice. J Neurophysiol 102:337-348.

Lundfald L, Restrepo CE, Butt SJ, Peng CY, Droho S, Endo T, Zeilhofer HU, Sharma K, Kiehn O (2007) Phenotype of V2-derived interneurons and their relationship to the axon guidance molecule EphA4 in the developing mouse spinal cord. Eur J Neurosci 26:2989-3002.

Lüthi A, Bal T, McCormick DA (1998) Periodicity of thalamic spindle waves is abolished by ZD 7288, a blocker of $\mathrm{I}_{\mathrm{h}}$. J Neurophysiol 79:3284-3289.

MacLean JN, Cowley KC, Schmidt BJ (1998) NMDA receptor-mediated oscillatory activity in the neonatal rat spinal cord is serotonin dependent. J Neurophysiol 79:2804-2808. 
Madriaga MA, McPhee LC, Chersa T, Christie KJ, Whelan PJ (2004) Modulation of locomotor activity by multiple 5-HT and dopaminergic receptor subtypes in the neonatal mouse spinal cord. J Neurophysiol 92:1566-1576.

McCormick DA, Pape HC (1990) Properties of a hyperpolarizationactivated cation current and its role in rhythmic oscillation in thalamic relay neurons. J Physiol 431:291-318.

McLean DL, Fan J, Higashijima S, Hale ME, Fetcho JR (2007) A topographic map of recruitment in spinal cord. Nature 446:71-75.

McLean DL, Masino MA, Koh IY, Lindquist WB, Fetcho JR (2008) Continuous shifts in the active set of spinal interneurons during changes in locomotor speed. Nat Neurosci 11:1419-1429.

Pearlstein E, Ben Mabrouk F, Pflieger JF, Vinay L (2005) Serotonin refines the locomotor related alternations in the in vitro neonatal rat spinal cord. Eur J Neurosci 21:1338-1346.

Peng CY, Yajima H, Burns CE, Zon LI, Sisodia SS, Pfaff SL, Sharma K (2007) Notch and MAML signaling drives Scl-dependent interneuron diversity in the spinal cord. Neuron 53:813-827.

Personius KE, Chang Q, Mentis GZ, O’Donovan MJ, Balice-Gordon RJ (2007) Reduced gap junctional coupling leads to uncorrelated motor neuron firing and precocious neuromuscular synapse elimination. Proc Natl Acad Sci U S A 104:11808-11813.

Richter SH, Garner JP, Würbel H (2009) Environmental standardization: cure or cause of poor reproducibility in animal experiments? Nat Methods 6:257-261.

Schmidt BJ, Jordan LM (2000) The role of serotonin in reflex modulation and locomotor rhythm production in the mammalian spinal cord. Brain Res Bull 53:689-710.

Shefchyk SJ, Jordan LM (1985) Motoneuron input-resistance changes during fictive locomotion produced by stimulation of the mesencephalic locomotor region. J Neurophysiol 54:1101-1108.

Stosiek C, Garaschuk O, Holthoff K, Konnerth A (2003) In vivo two-photon calcium imaging of neuronal networks. Proc Natl Acad Sci U S A 100:7319-7324.

Thoby-Brisson M, Telgkamp P, Ramirez JM (2000) The role of the hyperpolarization-activated current in modulating rhythmic activity in the isolated respiratory network of mice. J Neurosci 20:2994-3005.

Tresch MC, Kiehn O (2000) Motor coordination without action potentials in the mammalian spinal cord. Nat Neurosci 3:593-599.

Whelan P, Bonnot A, O'Donovan MJ (2000) Properties of rhythmic activity generated by the isolated spinal cord of the neonatal mouse. J Neurophysiol 84:2821-2833.

Wilson JM, Dombeck DA, Díaz-Ríos M, Harris-Warrick RM, Brownstone RM (2007) Two-photon calcium imaging of network activity in XFPexpressing neurons in the mouse. J Neurophysiol 97:3118-3125.

Zar JH (2000) Biostatistical analysis. Englewood Cliffs, NJ: Prentice-Hall.

Zhang W, Grillner S (2000) The spinal 5-HT system contributes to the generation of fictive locomotion in lamprey. Brain Res 879:188-192.

Zhang Y, Narayan S, Geiman E, Lanuza GM, Velasquez T, Shanks B, Akay T, Dyck J, Pearson K, Gosgnach S, Fan CM, Goulding M (2008) V3 spinal neurons establish a robust and balanced locomotor rhythm during walking. Neuron 60:84-96.

Zhong G, Díaz-Ríos M, Harris-Warrick RM (2006a) Serotonin modulates the properties of ascending commissural interneurons in the neonatal mouse spinal cord. J Neurophysiol 95:1545-1555.

Zhong G, Díaz-Ríos M, Harris-Warrick RM (2006b) Intrinsic and functional differences among commissural interneurons during fictive locomotion and serotonergic modulation in the neonatal mouse. J Neurosci 26:6509-6517.

Zhong G, Masino MA, Harris-Warrick RM (2007) Persistent sodium currents participate in fictive locomotion generation in neonatal mouse spinal cord. J Neurosci 27:4507-4518.

Zipfel WR, Williams RM, Webb WW (2003) Nonlinear magic: multiphoton microscopy in the biosciences. Nat Biotechnol 21:1369-1377. 